\title{
The Total Synthesis of Tubulysin D
}

Hillary M. Peltier, Jeffrey P. McMahon, Andrew W. Patterson, and Jonathan A. Ellman* Department of Chemistry, University of California, Berkeley, California 94720

jellman@berkeley.edu

\section{Supporting Information}

\section{Table of Contents}

$\begin{array}{ll}\text { I. General Methods } & \text { S2 }\end{array}$

II. Experimental Details and Compound Characterization S2 - S17

III. Spectral Comparison Table of Natural to Synthetic Tubulysin D $\quad$ S17 - S19

IV. ${ }^{1}$ H NMR Spectra for Compounds $3,4,6,7,8,14,15$, and $16 \quad$ S20 - S27

V. ${ }^{1}$ H NMR and ${ }^{13}$ C NMR Spectra for Tubulysin D $\quad$ S28 - S31

VI. ORTEP Diagram of $\mathbf{3}$ and bis-acylated adduct of $\mathbf{9}$ S32 - S33 
General Methods. Unless otherwise noted, all starting materials were obtained from commercial suppliers and used without further purification. THF, toluene, ether, and $\mathrm{CH}_{2} \mathrm{Cl}_{2}$ were dried over alumina under a nitrogen atmosphere. $i \mathrm{Pr}_{2} \mathrm{NH}$ and $i \operatorname{Pr}_{2} \mathrm{EtN}$ were distilled from $\mathrm{CaH}_{2}$ immediately prior to use. The concentration of commercially available solutions of $n$-butyllithium in hexanes was determined by titration with 2,6-ditert-butyl-4-methylphenol and 1,10-phenanthroline. Reactions were carried out in flameor oven-dried glassware under a $\mathrm{N}_{2}$ atmosphere. Extracts were dried over $\mathrm{Na}_{2} \mathrm{SO}_{4}$ and concentrated using a Buchi rotary evaporator under reduced pressure. Chromatography was carried out either with Merck $60 \AA 2$ 230-400 mesh silica gel or via normal-phase HPFC purification on a Biotage SP1 instrument (Charlottesville, VA) equipped with a Biotage Si flash column. IR spectra were recorded on a Nicolet Avatar 360 FTIR spectrometer equipped with an attenuated total reflectance accessory and only partial data are listed. ${ }^{1} \mathrm{H}$ NMR and ${ }^{13} \mathrm{C}$ NMR spectra were obtained at room temperature. Chemical shifts are expressed in ppm relative to solvent. Coupling constants are reported in $\mathrm{Hz}$. HPLC analyses of diastereomeric mixtures were performed on Diacel CHIRALCEL AD or AS ( 4.6 by $250 \mathrm{~mm}$ ) column with UV detection at 210 and $254 \mathrm{~nm}$. Elemental analyses and high-resolution mass spectra were performed by the University of California at Berkeley Micro-Mass Facility.

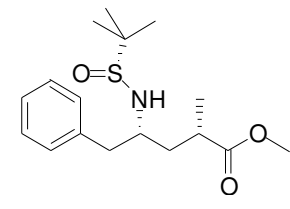

Amino ester 3. Diiodoethane was dissolved in ether and washed one time with saturated aqueous $\mathrm{Na}_{2} \mathrm{~S}_{2} \mathrm{O}_{3}$. The organic portion was separated, dried over $\mathrm{MgSO}_{4}$, filtered, and 
concentrated. A $0.2 \mathrm{M}$ solution of $\mathrm{SmI}_{2}$ was then prepared by adding diiodoethane (1.52 $\mathrm{g}, 5.40 \mathrm{mmol}, 1$ equiv) in deoxygenated THF to samarium powder (1.62 g, $10.8 \mathrm{mmol}, 2$ equiv). This mixture was stirred for $2 \mathrm{~h}$ at $\mathrm{rt}$, and then was used immediately. THF (4 mL, deoxygenated) was added to anhydrous $\mathrm{LiBr}(938 \mathrm{mg}, 10.8 \mathrm{mmol}) . \mathrm{SmI}_{2}(22.5 \mathrm{~mL}$, 0.2 $\mathrm{M}$ in $\mathrm{THF}$ ) was then added to the $\mathrm{LiBr}$ solution, and the mixture was stirred for 30 min. In a separate reaction vessel, deoxygenated THF $(3.5 \mathrm{~mL})$, a solution of sulfinyl imine $2^{1}$ in deoxygenated THF (0.5 M in THF, $\left.1.80 \mathrm{~mL}, 0.900 \mathrm{mmol}\right)$, methyl methacrylate $(0.481 \mathrm{~mL}, 4.50 \mathrm{mmol})$, and deoxygenated $\mathrm{H}_{2} \mathrm{O}(0.130 \mathrm{~mL}, 7.20 \mathrm{mmol})$ were combined. This solution was then cooled to $-78{ }^{\circ} \mathrm{C}$, and the $\mathrm{SmI}_{2}$ solution was transferred drop wise via cannula to the imine solution over $30 \mathrm{~min}$. The reaction mixture was stirred at $-78{ }^{\circ} \mathrm{C}$ for $12 \mathrm{~h}$. Saturated aqueous $\mathrm{Na}_{2} \mathrm{~S}_{2} \mathrm{O}_{3}(60 \mathrm{~mL})$ was then added, and the mixture was allowed to warm to room temperature. The mixture was extracted with EtOAc $(3 \times 100 \mathrm{~mL})$, and the combined organic portions were washed once with brine, dried over $\mathrm{MgSO}_{4}$, filtered, and concentrated. HPFC purification (97.5:2.5 hexanes: $i \mathrm{PrOH})$ provided $98.5 \mathrm{mg}$ of the pure major diastereomer $\mathbf{3}$ as a colorless oil. Mixed fractions were collected and purified a second time under the same conditions to provide another $61.1 \mathrm{mg}$ of pure major diastereomer $\mathbf{3}$ as a colorless oil for a combined yield of 55\%. HPLC analysis $(250 \mathrm{~mm} \times 4.6 \mathrm{~mm}$ Chiralcel AD column, detection at $210 \mathrm{~nm}, 1 \mathrm{~mL} / \mathrm{min}, 97: 3$ hexanes:iPrOH) of the unpurified material established an $80\left(t_{R}=20.9 \mathrm{~min}\right): 15\left(t_{R}=19.5 \mathrm{~min}\right): 3\left(t_{R}=14.7 \mathrm{~min}\right): 2\left(t_{R}=24.3\right.$ $\min$ ) ratio of diastereomers.

Major diastereomer: $[\alpha]_{\mathrm{D}}^{25}=+28.4^{\circ}\left(c=1, \mathrm{CHCl}_{3}\right)$ IR: $700,1047,1732,2951 \mathrm{~cm}^{-1} \cdot{ }^{1} \mathrm{H}$ $\operatorname{NMR}\left(500 \mathrm{MHz}, \mathrm{CDCl}_{3}\right) \delta 1.12(\mathrm{~d}, 3 \mathrm{H}, J=7.1 \mathrm{~Hz}), 1.18(\mathrm{~s}, 9 \mathrm{H}), 1.42(\mathrm{ddd}, 1 \mathrm{H}, J=4.1$, 
$10.3,14.3 \mathrm{~Hz}), 1.83(\mathrm{ddd}, 1 \mathrm{H}, J=3.9,10.3,14.2 \mathrm{~Hz}), 2.63-2.70(\mathrm{~m}, 1 \mathrm{H}), 2.98-3.01(\mathrm{~m}$, 2H), $3.23(\mathrm{~d}, 1 \mathrm{H}, \mathrm{J}=9.0 \mathrm{~Hz}), 3.50-3.57(\mathrm{~m}, 1 \mathrm{H}), 3.65(\mathrm{~s}, 3 \mathrm{H}), 7.21-7.25(\mathrm{~m}, 3 \mathrm{H}), 7.29-$ $7.32(\mathrm{~m}, 2 \mathrm{H}) .{ }^{13} \mathrm{C}$ NMR $\left(125 \mathrm{MHz}, \mathrm{CDCl}_{3}\right) \delta 18.1,22.9,36.4,39.4,43.0,51.8,55.9$, 56.3, 126.8, 128.7, 130.4, 136.9, 176.9. HRMS (FAB) calcd. for $\mathrm{C}_{17} \mathrm{H}_{28} \mathrm{NO}_{3} \mathrm{~S}(\mathrm{M}+\mathrm{H})$ : 326.1790. Found 326.1793.

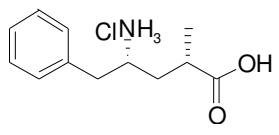

Tubuphenylalanine (4). $\mathrm{H}_{2} \mathrm{O}(3.07 \mathrm{~mL})$ was added to 3 (100 mg, $\left.0.307 \mathrm{mmol}\right)$, followed by conc. $\mathrm{HCl}(3.07 \mathrm{~mL})$, and the reaction mixture was heated to $100{ }^{\circ} \mathrm{C}$ for $1 \mathrm{~h}$. The mixture was then allowed to cool to room temperature and concentrated under vacuum. The resulting oil was triturated three times with $\mathrm{Et}_{2} \mathrm{O}$ to give $75.0 \mathrm{mg}(100 \%)$ of 4 as a white powder. $[\alpha]^{25}=+3.2^{\circ}(c=1, \mathrm{MeOH})$ IR: $701,740,1190,1705,2880$, $3076 \mathrm{~cm}^{-1}$. ${ }^{1} \mathrm{H}$ NMR $\left(500 \mathrm{MHz}, \mathrm{D}_{2} \mathrm{O}\right) \delta 1.07(\mathrm{~d}, 3 \mathrm{H}, J=7.0 \mathrm{~Hz}), 1.59-1.65(\mathrm{~m}, 1 \mathrm{H})$, 1.87-1.93 (m, 1H), 2.54-2.60 (m, 1H), $2.80(\mathrm{dd}, 1 \mathrm{H}, J=7.6,14.2 \mathrm{~Hz}), 2.93(\mathrm{dd}, 1 \mathrm{H}, J=$ 6.6, 14.2 Hz), 3.49 (app. quintet, $1 \mathrm{H}, J=6.9 \mathrm{~Hz}), 7.20(\mathrm{~d}, 2 \mathrm{H}, J=7.1 \mathrm{~Hz}), 7.25(\mathrm{t}, 1 \mathrm{H}, J$ $=7.3 \mathrm{~Hz}), 7.29(\mathrm{~m}, 2 \mathrm{H}) .{ }^{13} \mathrm{C} \mathrm{NMR}\left(125 \mathrm{MHz}, \mathrm{D}_{2} \mathrm{O}\right) \delta 17.3,36.1,36.5,39.0,51.9,128.2$, 129.8, 130.1, 136.1, 180.7. HRMS (FAB) calcd. for $\mathrm{C}_{12} \mathrm{H}_{18} \mathrm{NO}_{2}(\mathrm{M}+\mathrm{H})$ : 208.1341. Found 208.1338.

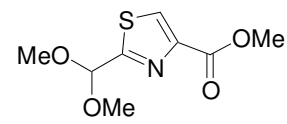

Methyl 2-(dimethoxymethyl)-4-thiazolecarboxylate. To a $0.5 \mathrm{M}$ solution of ethyl 2(diethoxymethyl)-4-thiazolecarboxylate ${ }^{2}(12.9 \mathrm{~g}, 49.7 \mathrm{mmol})$ in methanol (100 mL) was 
added $1 \mathrm{~mL}$ of concentrated $\mathrm{HCl}$. The solution was heated to reflux and stirred overnight. The reaction mixture was cooled to room temperature and concentrated under reduced pressure. The resulting oil was taken up in ethyl acetate and washed once with saturated aqueous $\mathrm{NaHCO}_{3}$. The organic layer was separated, dried over $\mathrm{MgSO}_{4}$, filtered, and concentrated under reduced pressure. The crude product was purified by column chromatography using a gradient elution system (90:10 to 40:60 hexanes/EtOAc) to deliver $9.38 \mathrm{~g}(87 \%)$ of product as a yellow oil. IR: 1721, 2836, $2954 \mathrm{~cm}^{-1} .{ }^{1} \mathrm{H}$ NMR $\left(400 \mathrm{MHz}, \mathrm{CDCl}_{3}\right): \delta 3.42$ (s, 6H), $3.93(\mathrm{~s}, 3 \mathrm{H}), 5.57(\mathrm{~s}, 1 \mathrm{H}), 8.19$ (s, 1H). ${ }^{13} \mathrm{C} \mathrm{NMR}$ (125 MHz, $\left.\mathrm{CDCl}_{3}\right): \delta 52.6,54.1,100.4,128.9,147.1,162.0,169.1$. HRMS (FAB) calcd. for $\mathrm{C}_{8} \mathrm{H}_{12} \mathrm{NO}_{4} \mathrm{~S}(\mathrm{M}+\mathrm{H}):$ 218.0487. Found 218.0491.

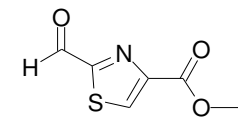

Methyl 2-formylthiazole-4-carboxylate (6). To a $0.5 \mathrm{M}$ solution of dimethyl acetal (10.6 g, $48.8 \mathrm{mmol})$ in acetone $(95 \mathrm{~mL})$ was added an equivalent volume of $1 \mathrm{M} \mathrm{HCl}$. The reaction mixture was stirred overnight at room temperature. The solvent was removed under reduced pressure, EtOAc $(75 \mathrm{~mL})$ was added, and the solution was washed with saturated aqueous $\mathrm{NaHCO}_{3}$. The aqueous layer was extracted twice with EtOAc $(50 \mathrm{~mL})$. The combined organic fractions were dried over $\mathrm{MgSO}_{4}$, filtered, and concentrated to give $6.69 \mathrm{~g}(80 \%)$ of $\mathbf{6}$ as a white solid. $\mathrm{mp} 116-117^{\circ} \mathrm{C}$. IR: 1678,1738 , $3134 \mathrm{~cm}^{-1} .{ }^{1} \mathrm{H}$ NMR $\left(400 \mathrm{MHz}, \mathrm{CDCl}_{3}\right) \delta 4.02(\mathrm{~s}, 3 \mathrm{H}), 8.53(\mathrm{~d}, 1 \mathrm{H}, \mathrm{J}=1.2 \mathrm{~Hz}), 10.07(\mathrm{~d}$, $1 \mathrm{H}, \mathrm{J}=1.2 \mathrm{~Hz}) .{ }^{13} \mathrm{C} \mathrm{NMR}\left(125 \mathrm{MHz}, \mathrm{CDCl}_{3}\right) \delta 53.1,133.4,149.3,161.2,166.3,183.7$. Anal. calcd. for $\mathrm{C}_{6} \mathrm{H}_{5} \mathrm{NO}_{3} \mathrm{~S}: \mathrm{C}, 42.10 ; \mathrm{H}, 2.94 ; \mathrm{N}, 8.18$. Found: C, 42.11; H, 2.80; N, 8.15 . 


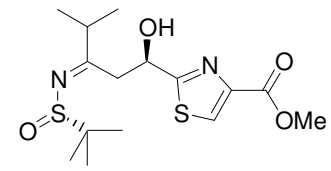

Imino alcohol 7. A $0.5 \mathrm{M}$ solution of diisopropylamine (5.59 mL, $66.2 \mathrm{mmol})$ in $\mathrm{Et}_{2} \mathrm{O}$ $(132 \mathrm{~mL})$ was cooled to $0{ }^{\circ} \mathrm{C}$ and $n$-butyllithium $(32.3 \mathrm{~mL}, 60.7 \mathrm{mmol}, 1.88 \mathrm{M}$ solution in hexanes) was added. The solution was stirred for $20 \mathrm{~min}$ at $0{ }^{\circ} \mathrm{C}$ and then cooled to $78^{\circ} \mathrm{C}$. A solution of sulfinyl imine $5^{1}(10.5 \mathrm{~g}, 55.2 \mathrm{mmol})$ in $\mathrm{Et}_{2} \mathrm{O}(116 \mathrm{~mL})$ was added, and the reaction mixture was stirred for $30 \mathrm{~min}$ at $-78{ }^{\circ} \mathrm{C}$. Chlorotitanium triisopropoxide (26.4 $\mathrm{mL}, 110 \mathrm{mmol}$ ) was then added, and the reaction mixture was stirred for an additional $45 \mathrm{~min}$ at $-78{ }^{\circ} \mathrm{C}$. Aldehyde $6(6.29 \mathrm{~g}, 36.8 \mathrm{mmol})$ was added in one portion, and the solution was stirred at $-78{ }^{\circ} \mathrm{C}$ for $12 \mathrm{~h}$. The solution was neutralized using a 4:1 (v/v) solution of THF/AcOH and allowed to warm to room temperature. Water $(50 \mathrm{~mL})$ was added, and the resulting mixture was filtered through celite, washing the filter cake thoroughly with EtOAc. The solution was washed with brine, dried over $\mathrm{MgSO}_{4}$, filtered, and concentrated under reduced pressure. HPFC purification (70:30 to 0:100 hexanes:ether) produced $12.0 \mathrm{~g}(90 \%)$ of the pure major diastereomer $\mathbf{7}$ as a colorless oil and $1.19 \mathrm{~g}(9 \%)$ of the minor diastereomer as a yellow oil. Major diastereomer (7): $[\alpha]^{25}{ }_{\mathrm{D}}=+69.6^{\circ}\left(c=1, \mathrm{CHCl}_{3}\right)$. IR: 1031, 1094, 1212, 1240, 1624, 1724, $2967 \mathrm{~cm}^{-1} .{ }^{1} \mathrm{H}$ NMR $\left(500 \mathrm{MHz}, \mathrm{CDCl}_{3}\right) \delta 1.19(\mathrm{~m}, 6 \mathrm{H}), 1.28(\mathrm{~s}, 9 \mathrm{H}), 2.84$ (sep, $1 \mathrm{H}, J=6.7 \mathrm{~Hz}), 3.26-3.35(\mathrm{~m}, 2 \mathrm{H}), 3.92(\mathrm{~s}, 3 \mathrm{H}), 5.11(\mathrm{~m}, 1 \mathrm{H}), 6.61(\mathrm{~d}, 1 \mathrm{H}, J=8$ $\mathrm{Hz}), 8.12(\mathrm{~s}, 1 \mathrm{H}) .{ }^{13} \mathrm{C} \mathrm{NMR}\left(125 \mathrm{MHz}, \mathrm{CDCl}_{3}\right) \delta 20.3,21.2,22.6,39.1,44.0,52.6,58.7$, 67.7, 128.2, 147.1, 162.1, 178.6, 187.8. HRMS (FAB) calcd. for $\mathrm{C}_{15} \mathrm{H}_{25} \mathrm{~N}_{2} \mathrm{O}_{4} \mathrm{~S}_{2}(\mathrm{M}+\mathrm{H})$ : 361.1256. Found 361.1256. 
Minor diastereomer: ${ }^{1} \mathrm{H}$ NMR $\left(300 \mathrm{MHz}, \mathrm{CDCl}_{3}\right) \delta 0.81(\mathrm{~d}, 3 \mathrm{H}, J=6.5 \mathrm{~Hz}), 0.99(\mathrm{~d}, 3 \mathrm{H}$, $J=6.8 \mathrm{~Hz}), 1.31(\mathrm{~s}, 9 \mathrm{H}), 1.60-1.70(\mathrm{~m}, 1 \mathrm{H}), 3.42(\mathrm{dd}, 1 \mathrm{H}, J=3.5,12.6 \mathrm{~Hz}), 3.54(\mathrm{dd}, 1 \mathrm{H}$, $J=5.2,12.6 \mathrm{~Hz}), 3.96(\mathrm{~s}, 3 \mathrm{H}), 5.33-5.37(\mathrm{~m}, 1 \mathrm{H}), 6.65(\mathrm{~s}, 1 \mathrm{H}), 8.14(\mathrm{~s}, 1 \mathrm{H})$.

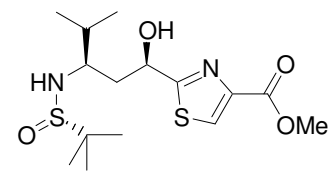

Amino alcohol 8. A $0.4 \mathrm{M}$ solution of imine $7(207 \mathrm{mg}, 0.570 \mathrm{mmol})$ in THF $(1.38 \mathrm{~mL})$ was cooled to $-78^{\circ} \mathrm{C}$. $\mathrm{Ti}(\mathrm{OEt})_{4}(0.266 \mathrm{~mL}, 1.14 \mathrm{mmol})$ was added, followed by $\mathrm{NaBH}_{4}$ ( $86.0 \mathrm{mg}, 2.28 \mathrm{mmol}$ ), and the solution was allowed to slowly warm to $-45^{\circ} \mathrm{C}$ over 30 min. The reaction mixture was stirred for $1 \mathrm{~h}$ at $-45^{\circ} \mathrm{C}$, then $\mathrm{MeOH}$ was added slowly, keeping the internal temperature of the reaction below $-30^{\circ} \mathrm{C}$, until gas evolution ceased. The solution was warmed to room temperature and water $(10 \mathrm{~mL})$ was added. The resulting suspension was filtered through a pad of celite, washing the filter cake thoroughly with EtOAc. The solution was washed once with brine, dried, filtered, and concentrated. HPLC analysis (250 mm x $4.6 \mathrm{~mm}$ ChiralPak AS column, detection at 254 $\mathrm{nm}, 1 \mathrm{~mL} / \mathrm{min}, 98: 2$ hexanes:EtOH$)$ on the unpurified material established a $91\left(\mathrm{t}_{\mathrm{R}}=6.5\right.$ $\min )$ to $9\left(t_{R}=14.3 \mathrm{~min}\right)$ ratio of diastereomers. The product was then purified via column chromatography using EtOAc as the eluent to provide $182 \mathrm{mg}(88 \%)$ of the pure major diastereomer $\mathbf{8}$ as a colorless oil.

Major diastereomer $(\mathbf{8}):[\alpha]^{25}{ }_{\mathrm{D}}=+116.6^{\circ}\left(c=1, \mathrm{CHCl}_{3}\right)$ IR: $751,1027,1216,1726$, $2959 \mathrm{~cm}^{-1} .{ }^{1} \mathrm{H}$ NMR $\left(500 \mathrm{MHz}, \mathrm{CDCl}_{3}\right): \delta 0.90(\mathrm{~d}, 3 \mathrm{H}, J=6.7 \mathrm{~Hz}), 0.93(\mathrm{~d}, 3 \mathrm{H}, J=6.8$ $\mathrm{Hz}), 1.28(\mathrm{~s}, 9 \mathrm{H}), 1.66-1.73(\mathrm{~m}, 1 \mathrm{H}), 1.88-1.94(\mathrm{~m}, 1 \mathrm{H}), 2.25-2.31(\mathrm{~m}, 1 \mathrm{H}), 3.30(\mathrm{~d}, 1 \mathrm{H}$, $J=8.4 \mathrm{~Hz}), 3.40-3.46(\mathrm{~m}, 1 \mathrm{H}), 3.93(\mathrm{~s}, 3 \mathrm{H}), 5.15-5.20(\mathrm{~m}, 1 \mathrm{H}), 5.49(\mathrm{~d}, 1 \mathrm{H}, J=6.7 \mathrm{~Hz})$, 
$8.12(\mathrm{~s}, 1 \mathrm{H}) .{ }^{13} \mathrm{C}$ NMR $\left(125 \mathrm{MHz}, \mathrm{CDCl}_{3}\right): \delta 17.5,19.7,23.2,34.1,40.9,52.5,56.5$, 58.7, 67.9, 127.8, 146.7, 162.2, 178.4. HRMS (FAB) calcd. for $\mathrm{C}_{15} \mathrm{H}_{27} \mathrm{~N}_{2} \mathrm{O}_{4} \mathrm{~S}_{2}(\mathrm{M}+\mathrm{H})$ : 363.1412. Found 363.1410.

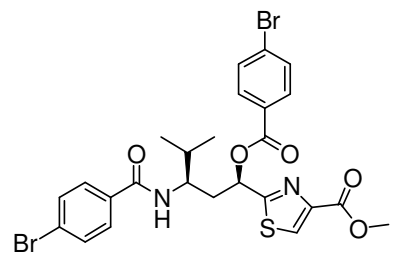

Bis-acylation adduct of 9. $4.0 \mathrm{~N} \mathrm{HCl}$ in dioxane $(2.85 \mathrm{~mL}, 11.4 \mathrm{mmol})$ was added to a $0.16 \mathrm{M}$ solution of $\mathbf{8}(0.413 \mathrm{~g}, 1.14 \mathrm{mmol})$ in $\mathrm{MeOH}$. The reaction mixture was stirred for $2 \mathrm{~h}$ at $\mathrm{rt}$ and concentrated to yield amino alcohol 9 as a white solid. A $0.020 \mathrm{M}$ solution of amino alcohol 9 (200 mg, $1.14 \mathrm{mmol}, 1.0$ equiv) in $\mathrm{CH}_{2} \mathrm{Cl}_{2}(5.70 \mathrm{~mL})$ was cooled to $0{ }^{\circ} \mathrm{C}$. Triethylamine $(0.480 \mathrm{~mL}, 3.42 \mathrm{mmol}, 3.0$ equiv), para-bromobenzoyl chloride (1.20 g, $5.69 \mathrm{mmol}, 5.0$ equiv), and a catalytic amount of DMAP were added. The reaction mixture was allowed to warm to rt, and stirring was continued for $18 \mathrm{~h}$. Brine $(50 \mathrm{~mL})$ was added to the reaction mixture, and the aqueous layer was extracted with EtOAc $(2 \times 75 \mathrm{~mL})$. The combined organic portions were dried, filtered, and concentrated. Column chromatography (99:1 to $95: 5 \mathrm{MeOH}: \mathrm{CH}_{2} \mathrm{Cl}_{2}$ ) afforded $52.0 \mathrm{mg}$ (7\%) of the desired product as a colorless crystalline solid. Vapor diffusion with $\mathrm{CH}_{2} \mathrm{Cl}_{2}$ /pentane yielded X-ray quality crystals. $[\alpha]^{23}{ }_{\mathrm{D}}=+34.3^{\circ}\left(c=1, \mathrm{CHCl}_{3}\right) . \mathrm{mp} 148-$ $154{ }^{\circ} \mathrm{C}$. IR: $1483,1537,1590,1640,1727,2961,3355 \mathrm{~cm}^{-1} .{ }^{1} \mathrm{H}$ NMR $(500 \mathrm{MHz}$, $\left.\mathrm{CDCl}_{3}\right): \delta 0.963(\mathrm{~d}, 6 \mathrm{H}, J=6.9 \mathrm{~Hz}), 1.88-1.95(\mathrm{~m}, 1 \mathrm{H}), 2.44($ apparent t, $2 \mathrm{H}, J=7.0 \mathrm{~Hz})$, $3.93(\mathrm{~s}, 3 \mathrm{H}), 4.27-4.33(\mathrm{~m}, 1 \mathrm{H}), 6.29(\mathrm{~d}, 1 \mathrm{H}, J=9.5 \mathrm{~Hz}), 6.36-6.39(\mathrm{~m}, 1 \mathrm{H}), 7.48(\mathrm{~d}, 2 \mathrm{H}$, $J=9.2 \mathrm{~Hz}), 7.55-7.57(\mathrm{~m}, 4 \mathrm{H}), 7.90(\mathrm{~d}, 2 \mathrm{H}, J=8.4 \mathrm{~Hz}), 8.10(\mathrm{~s}, 1 \mathrm{H}) .{ }^{13} \mathrm{C} \mathrm{NMR}(125$ 
$\left.\mathrm{MHz}, \mathrm{CDCl}_{3}\right): \delta 18.6,19.1,33.0,37.3,51.4,52.9,71.6,126.3,128.2,128.3,128.8,129.2$, $131.7,132.0,132.2,133.5,147.1,161.8,165.0,166.6,171.3 . \mathrm{MS}(\mathrm{FAB}): \mathrm{m} / z 625\left(\mathrm{MH}^{+}\right)$. Anal. calcd. for $\mathrm{C}_{25} \mathrm{H}_{24} \mathrm{Br}_{2} \mathrm{~N}_{2} \mathrm{O}_{5} \mathrm{~S}: \mathrm{C}, 48.09 ; \mathrm{H}, 3.87 ; \mathrm{N}, 4.49$. Found: C, 47.83; H, 3.99; $\mathrm{N}, 4.26$.

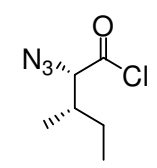

Acid chloride 10. Oxalyl chloride (2.44 mL, $28.0 \mathrm{mmol})$ and DMF (0.464 mL, 5.97 mmol) were added to a $0.024 \mathrm{M}$ solution of azido isoleucine ${ }^{3}(0.938 \mathrm{~g}, 5.97 \mathrm{mmol})$ in hexanes. The reaction mixture was stirred at $\mathrm{rt}$ for $1 \mathrm{~h}$, filtered, and concentrated. Acid chloride 10 was used immediately without further purification.

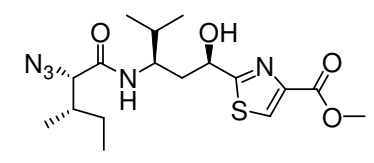

Compound 11. $4.0 \mathrm{~N} \mathrm{HCl}$ in dioxane $(9.33 \mathrm{~mL}, 37.3 \mathrm{mmol})$ was added to a $0.16 \mathrm{M}$ solution of $8(1.35 \mathrm{~g}, 3.73 \mathrm{mmol})$ in $\mathrm{MeOH}$. The reaction mixture was stirred for $2 \mathrm{~h}$ at rt and concentrated to yield amino alcohol $\mathbf{9}$ as a white solid. A $0.10 \mathrm{M}$ solution of amino alcohol 9 (1.10 g, $3.73 \mathrm{mmol}, 1.0$ equiv) in $\mathrm{CH}_{2} \mathrm{Cl}_{2}(37.3 \mathrm{~mL})$ was cooled to $0{ }^{\circ} \mathrm{C}$. Diisopropylethylamine (3.25 mL, $18.7 \mathrm{mmol}, 5.0$ equiv) and acid chloride 10 (1.05 g, $5.97 \mathrm{mmol}, 1.6$ equiv) were added. The reaction mixture was allowed to warm to $\mathrm{rt}$ and stirred for $18 \mathrm{~h}$. Brine $(50 \mathrm{~mL})$ was added to the reaction mixture, and the aqueous layer was extracted with EtOAc $(2 \times 75 \mathrm{~mL})$. The combined organic portions were dried, filtered, and concentrated. Column chromatography (1:1 hexanes/EtOAc) afforded 1.37 g (93\%) of $\mathbf{1 1}$ as a white solid. $[\alpha]^{23}=+18.7^{\circ}\left(c=1, \mathrm{CHCl}_{3}\right) . \mathrm{mp} 137.3-139.5^{\circ} \mathrm{C}$. IR: 
1650, 1732, 2102, 2964, $3279 \mathrm{~cm}^{-1} .{ }^{1} \mathrm{H}$ NMR (500 MHz, $\left.\mathrm{CDCl}_{3}\right): \delta 0.906(\mathrm{t}, 3 \mathrm{H}, J=7.3$ $\mathrm{Hz}), 0.940(\mathrm{~d}, 3 \mathrm{H}, J=6.9 \mathrm{~Hz}), 0.950(\mathrm{~d}, 3 \mathrm{H}, J=6.7 \mathrm{~Hz}), 1.06(\mathrm{~d}, 3 \mathrm{H}, J=6.9 \mathrm{~Hz}), 1.26-$ $1.32(\mathrm{~m}, 1 \mathrm{H}), 1.37-1.42(\mathrm{~m}, 1 \mathrm{H}), 1.73-1.88(\mathrm{~m}, 2 \mathrm{H}), 2.10-2.19(\mathrm{~m}, 2 \mathrm{H}), 3.92(\mathrm{~s}, 3 \mathrm{H}), 3.97$ $(\mathrm{m}, 1 \mathrm{H}), 4.01(\mathrm{~d}, 1 \mathrm{H}, J=3.5 \mathrm{~Hz}), 4.84-4.88(\mathrm{~m}, 1 \mathrm{H}), 5.27(\mathrm{~d}, 1 \mathrm{H}, J=4.6 \mathrm{~Hz}), 6.5(\mathrm{~d}, 1 \mathrm{H}$, $J=9.3 \mathrm{~Hz}), 8.13(\mathrm{~s}, 1 \mathrm{H}) .{ }^{13} \mathrm{C} \mathrm{NMR}\left(125 \mathrm{MHz}, \mathrm{CDCl}_{3}\right): \delta 11.9,16.3,18.6,20.0,24.4$, 32.1, 38.9, 41.4, 51.9, 52.7, 69.0, 69.9, 127.9, 146.8, 162.3, 171.1, 176.5. MS (FAB): $m / z$ $398\left(\mathrm{MH}^{+}\right)$. Anal. calcd. for $\mathrm{C}_{17} \mathrm{H}_{27} \mathrm{~N}_{5} \mathrm{O}_{4} \mathrm{~S}: \mathrm{C}, 51.37 ; \mathrm{H}, 6.85 ; \mathrm{N}, 17.62$. Found: $\mathrm{C}, 51.00$; H, 7.04; N, 17.26.

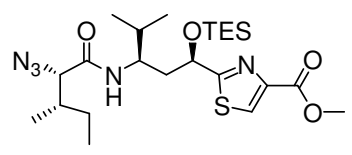

Compound 12. A $0.20 \mathrm{M}$ solution of amide 11 ( $0.650 \mathrm{~g}, 1.64 \mathrm{mmol}, 1.0$ equiv) in $\mathrm{CH}_{2} \mathrm{Cl}_{2}(8.18 \mathrm{~mL})$ was cooled to $0{ }^{\circ} \mathrm{C}$. 2,6-Lutidine $(0.950 \mathrm{~mL}, 8.18 \mathrm{mmol}, 5.0$ equiv $)$ and triethylsilyl trifluoromethanesulfonate $(0.930 \mathrm{~mL}, 4.00 \mathrm{mmol}, 2.4$ equiv $)$ were added. The reaction mixture was allowed to warm to $\mathrm{rt}$ over 1 hour and then stirred at $\mathrm{rt}$ for an additional hour. Brine $(50 \mathrm{~mL})$ was added to the reaction mixture, and the aqueous layer was extracted with EtOAc $(2 \times 75 \mathrm{~mL})$. The combined organic portions were dried, filtered, and concentrated under reduced pressure. Column chromatography (3:1 hexanes/EtOAc) afforded $820 \mathrm{mg}(98 \%)$ of $\mathbf{1 2}$ as a white solid. $[\alpha]^{23}{ }_{\mathrm{D}}=+22.6^{\circ}(c=1$, $\left.\mathrm{CHCl}_{3}\right) . \operatorname{mp} 48-50{ }^{\circ} \mathrm{C} . \mathrm{IR}: 1522,1678,1736,2110,2877,2960,3347 \mathrm{~cm}^{-1} .{ }^{1} \mathrm{H} \mathrm{NMR}$ $\left(500 \mathrm{MHz}, \mathrm{CDCl}_{3}\right): \delta 0.586-0.638(\mathrm{~m}, 6 \mathrm{H}), 0.798(\mathrm{~d}, 3 \mathrm{H}, J=7.2 \mathrm{~Hz}), 0.839(\mathrm{~d}, 3 \mathrm{H}, J=$ $7.0 \mathrm{~Hz}), 0.872(\mathrm{t}, 3 \mathrm{H}, J=7.5 \mathrm{~Hz}), 0.904(\mathrm{t}, 9 \mathrm{H}, J=8.0 \mathrm{~Hz}), 1.01(\mathrm{~d}, 3 \mathrm{H}, J=7.0 \mathrm{~Hz})$, 1.19-1.29 (m, 1H), 1.38-1.46 (m, 1H), 1.85-1.92 (m, 3H), 2.05-2.12 (m, 1H), $3.83(\mathrm{~d}, 1 \mathrm{H}$, $J=4.3 \mathrm{~Hz}), 3.91(\mathrm{~s}, 3 \mathrm{H}), 3.94-3.99(\mathrm{~m}, 1 \mathrm{H}), 5.11(\mathrm{t}, 1 \mathrm{H}, J=5.7 \mathrm{~Hz}), 6.63(\mathrm{~d}, 1 \mathrm{H}, J=8.5$ 
$\mathrm{Hz}), 8.10(\mathrm{~s}, 1 \mathrm{H}) .{ }^{13} \mathrm{C} \mathrm{NMR}\left(125 \mathrm{MHz}, \mathrm{CDCl}_{3}\right): \delta 4.9,7.0,11.8,16.3,17.8,18.6,24.4$, $31.9,38.6,40.2,51.5,52.7,70.4,70.9,127.9,146.7,162.1,168.6,178.4$. MS (FAB): $m / z$ $512\left(\mathrm{MH}^{+}\right)$. Anal. calcd. for $\mathrm{C}_{23} \mathrm{H}_{41} \mathrm{~N}_{5} \mathrm{O}_{4} \mathrm{SSi}: \mathrm{C}, 53.98 ; \mathrm{H}, 8.08 ; \mathrm{N}, 13.69$. Found: $\mathrm{C}$, 54.14; H, 8.35; N, 13.65 .

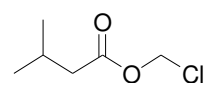

Chloromethyl ester. Chloromethyl ester was prepared following the literature procedure. ${ }^{4}{ }^{1} \mathrm{H}$ NMR $\left(500 \mathrm{MHz}, \mathrm{CDCl}_{3}\right): \delta 0.97(\mathrm{~d}, 6 \mathrm{H}, J=7.0 \mathrm{~Hz}), 2.14(\mathrm{~m}, 1 \mathrm{H}), 2.26$ $(\mathrm{d}, 2 \mathrm{H}, J=7.3 \mathrm{~Hz}), 5.70(\mathrm{~s}, 2 \mathrm{H}) .{ }^{13} \mathrm{C}$ NMR $\left(125 \mathrm{MHz}, \mathrm{CDCl}_{3}\right): \delta 22.6,25.9,43.2,68.8$, 171.4. ${ }^{1}$ H NMR shifts correspond with the literature data. ${ }^{4}$

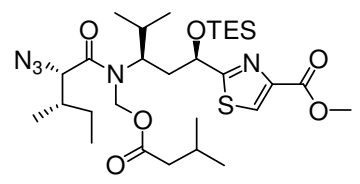

Compound 13. A $0.30 \mathrm{M}$ solution of amide $12(200 \mathrm{mg}, 0.390 \mathrm{mmol}, 1.0$ equiv) in THF $(1.3 \mathrm{~mL})$ was cooled to $-45^{\circ} \mathrm{C}$ and KHMDS $(0.934 \mathrm{~mL}, 0.469 \mathrm{mmol}, 0.50 \mathrm{M}$ in toluene, 1.2 equiv) was added. The resulting mixture was stirred for 20 minutes at $-45^{\circ} \mathrm{C}$. Chloromethyl ester (176 mg, $1.17 \mathrm{mmol}, 3.0$ equiv) was added, and the reaction mixture was allowed to warm to rt over $4.5 \mathrm{~h}$ at which time the reaction was quenched with $\mathrm{MeOH}(1.0 \mathrm{~mL})$. The crude product was diluted with EtOAc $(75 \mathrm{~mL})$ and washed with brine $(50 \mathrm{~mL})$. The aqueous layer was extracted with EtOAc $(2 \times 75 \mathrm{~mL})$. The organic portions were dried, filtered, and concentrated. The crude product was placed under reduced pressure $(0.05 \mathrm{~mm} \mathrm{Hg})$ for $18 \mathrm{~h}$ to remove the excess chloromethyl ester. Column chromatography (2:1 hexanes/EtOAc) yielded $178 \mathrm{mg}$ (73\%) of $\mathbf{1 3}$ as a colorless 
oil. $[\alpha]^{23}{ }_{\mathrm{D}}=+22.4^{\circ}\left(c=1, \mathrm{CHCl}_{3}\right)$. IR: $1673,1740,2100,2877,2961 \mathrm{~cm}^{-1} .{ }^{1} \mathrm{H} \mathrm{NMR}$ (500 MHz, $\left.\mathrm{CDCl}_{3}\right): \delta$ 0.592-0.676 (m, 6H), 0.851-0.948 (m, 27H), 1.23-1.29 (m, 1H), $1.67-1.76(\mathrm{~m}, 2 \mathrm{H}), 2.06-2.21(\mathrm{~m}, 4 \mathrm{H}), 2.29-2.32(\mathrm{~m}, 2 \mathrm{H}), 3.52(\mathrm{~d}, 1 \mathrm{H}, J=9.7 \mathrm{~Hz}), 3.91$ (s, 3H), 4.18-4.45 (br s, 1H), 4.97-5.05 (m, 1H), 5.39 (s, 2H), 8.10 (s, 1H). ${ }^{13} \mathrm{C}$ NMR $\left(125 \mathrm{MHz}, \mathrm{CDCl}_{3}\right): \delta 5.1,7.1,10.8,16.0,17.9,19.8,20.4,22.6,22.7,25.3,25.8,31.5$, $35.2,41.1,43.4,43.5,52.6,63.9,71.2,127.8,146.9,162.1,171.3,172.8,177.9$. MS (FAB): $m / z 632\left(\mathrm{MLi}^{+}\right)$. Anal. calcd. for $\mathrm{C}_{29} \mathrm{H}_{51} \mathrm{~N}_{5} \mathrm{O}_{6} \mathrm{SSi}$ C, $55.65 ; \mathrm{H}, 8.21 ; \mathrm{N}, 11.19$. Found: C, 55.54; H, 8.31; N, 11.14 .

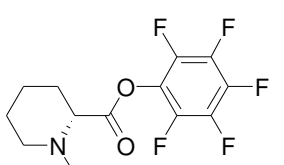

Pentafluorophenyl ester of Mep. To a $0.87 \mathrm{M}$ solution of D-pipecolinic acid (500 mg, $3.87 \mathrm{mmol}, 1.0$ equiv) in $\mathrm{MeOH}(4.45 \mathrm{~mL})$ was added a $40 \%$ aqueous solution of paraformaldehyde (233 mg, $7.74 \mathrm{mmol}, 2.0$ equiv) followed by Pd/C (110 mg). The reaction mixture was stirred for $24 \mathrm{~h}$ under a hydrogen atmosphere. Another 2.0 equiv of paraformaldehyde was added and the reaction mixture was stirred for an additional $18 \mathrm{~h}$ under a hydrogen atmosphere. The reaction mixture was filtered through celite, with washing of the filter pad with $\mathrm{MeOH}$. The filtrate was concentrated under reduced pressure to yield $491 \mathrm{mg}(89 \%)$ of desired product as a white solid. To a $0.4 \mathrm{M}$ solution of D- $N$-methyl pipecolinic acid (172 mg, $1.20 \mathrm{mmol}, 1.0$ equiv) in EtOAc (3.0 mL, filtered through a plug of activated alumina) were added pentafluorophenol (243 mg, 1.32 mmol, 1.1 equiv) and DCC (272 $\mathrm{mg}, 1.32 \mathrm{mmol}, 1.1$ equiv). The reaction mixture was 
stirred for $21 \mathrm{~h}$ at rt, at which time it was filtered, washing with $1.0 \mathrm{~mL}$ of EtOAc. The activated ester was used immediately without further purification or concentration.

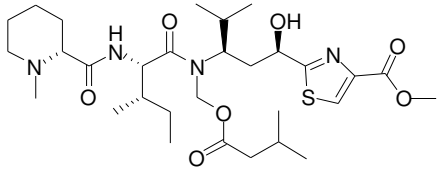

Compound 14. $\mathrm{Pd} / \mathrm{C}(10 \mathrm{wt} \%, 0.138 \mathrm{~g})$ and azide compound 13 (250 mg, $0.399 \mathrm{mmol})$ were added to a $0.3 \mathrm{M}$ solution of the activated ester of $\mathrm{D}-\mathrm{N}$-methyl pipecolinic acid in $4.0 \mathrm{~mL}$ of EtOAc. The reaction mixture was stirred under a hydrogen atmosphere for 27 h. The reaction mixture was filtered through a plug of celite, with washing of the filter pad with EtOAc. HPFC purification (0:100 to 1:99 MeOH:EtOAc) provided $268 \mathrm{mg}$ $(92 \%)$ of the coupled product.

A $0.02 \mathrm{M}$ solution of the coupled product in deoxygenated $\mathrm{AcOH} / \mathrm{H}_{2} \mathrm{O} / \mathrm{THF}(13 \mathrm{~mL}$, $3: 1: 1, \mathrm{v} / \mathrm{v} / \mathrm{v})$ was stirred at $\mathrm{rt}$ for $36 \mathrm{~h}$. Toluene $(50 \mathrm{~mL})$ was added and the solution was concentrated. HPFC purification (0:100 to 5:95 MeOH/EtOAc) afforded $189 \mathrm{mg}(85 \%)$ of 14. $[\alpha]^{23}{ }_{\mathrm{D}}=+2.20^{\circ}(c=1, \mathrm{MeOH})$. IR: $1505,1653,1739,2872,2934,2960,3360$ $\mathrm{cm}^{-1} .{ }^{1} \mathrm{H}$ NMR $(500 \mathrm{MHz}, \mathrm{MeOD}): \delta 0.72(\mathrm{~d}, 3 \mathrm{H}, J=6.5 \mathrm{~Hz}), 0.81(\mathrm{t}, 3 \mathrm{H}, J=7.3 \mathrm{~Hz})$, $0.84(\mathrm{~d}, 3 \mathrm{H}, J=6.7 \mathrm{~Hz}), 0.85(\mathrm{~d}, 3 \mathrm{H}, J=6.7 \mathrm{~Hz}), 0.90(\mathrm{~d}, 6 \mathrm{H}, J=6.5 \mathrm{~Hz}), 1.10-1.24(\mathrm{~m}$, $4 \mathrm{H}), 1.42-1.57(\mathrm{~m}, 4 \mathrm{H}), 1.66(\mathrm{~d}, 2 \mathrm{H}, J=9.6 \mathrm{~Hz}), 1.85-2.03(\mathrm{~m}, 4 \mathrm{H}), 2.10(\mathrm{~s}, 3 \mathrm{H}), 2.20-$ $2.35(\mathrm{~m}, 2 \mathrm{H}), 2.50(\mathrm{~d}, 1 \mathrm{H}, J=2.5 \mathrm{~Hz}), 2.83(\mathrm{~d}, 1 \mathrm{H}, J=12 \mathrm{~Hz}), 3.80(\mathrm{~s}, 3 \mathrm{H}), 4.51(\mathrm{~d}, 1 \mathrm{H}$, $J=9.5 \mathrm{~Hz}), 4.52(\mathrm{~m}, 1 \mathrm{H}), 4.74(\mathrm{~m}, 1 \mathrm{H}), 5.34(\mathrm{~d}, 1 \mathrm{H}, J=12 \mathrm{~Hz}), 6.10(\mathrm{~d}, 1 \mathrm{H}, J=12 \mathrm{~Hz})$, $8.21(\mathrm{~s}, 1 \mathrm{H}) .{ }^{13} \mathrm{C}$ NMR $(125 \mathrm{MHz}, \mathrm{MeOD}): \delta 11.5,17.1,21.6,23.62,23.65,25.1,26.8$, $26.98,27.5,32.4,38.39,38.43,40.08,40.14,45.1,45.5,53.4,56.1,57.5,70.5,71.2$, 
130.0, 148.5, 164.11, 164.14, 174.6, 176.4, 180.8. MS (FAB): $m / z 611\left(\mathrm{MH}^{+}\right)$. HRMS

(FAB) calcd for $\mathrm{C}_{30} \mathrm{H}_{51} \mathrm{~N}_{4} \mathrm{O}_{7} \mathrm{~S}(\mathrm{M}+\mathrm{H})$ : 611.3486. Found: 611.3478.

In order to assure that no epimerization occurred during the activated ester formation and coupling, L- $N$-methyl pipecolinic acid was synthesized and coupled to compound $\mathbf{1 3}$, followed by TES deprotection, as described above. The characterization data for this compound is as follows: ${ }^{1} \mathrm{H}$ NMR $(500 \mathrm{MHz}, \mathrm{MeOD}): \delta 0.69(\mathrm{~d}, 3 \mathrm{H}, J=6.6 \mathrm{~Hz}), 0.81(\mathrm{t}$, $3 \mathrm{H}, J=7.8 \mathrm{~Hz}), 0.83(\mathrm{~d}, 3 \mathrm{H}, J=6.9 \mathrm{~Hz}), 0.84(\mathrm{~d}, 3 \mathrm{H}, J=6.7 \mathrm{~Hz}), 0.89(\mathrm{~d}, 6 \mathrm{H}, J=6.4$ Hz), 1.09-1.20 (m, 2H), 1.34-1.55 (m, 5H), 1.58-1.66 (m, 2H), 1.82-2.00 (m, 5H), $2.06(\mathrm{~s}$, $3 \mathrm{H}), 2.19-2.32(\mathrm{~m}, 2 \mathrm{H}), 2.43(\mathrm{~d}, 1 \mathrm{H}, J=11.5 \mathrm{~Hz}), 2.82(\mathrm{~d}, 1 \mathrm{H}, J=11.0 \mathrm{~Hz}), 3.79(\mathrm{~s}, 3 \mathrm{H})$, $4.53(\mathrm{~d}, 1 \mathrm{H}, J=9.5 \mathrm{~Hz}), 4.72(\mathrm{~m}, 2 \mathrm{H}), 5.33(\mathrm{~d}, 1 \mathrm{H}, J=12.5 \mathrm{~Hz}), 6.05(\mathrm{~d}, 1 \mathrm{H}, J=12.5$ $\mathrm{Hz}), 8.20$ (s, 1H). ${ }^{13} \mathrm{C}$ NMR (125 MHz, MeOD): $\delta$ 11.5, 17.1, 21.3, 21.6, 23.5, 23.63, $23.65,25.2,26.7,27.1,27.4,32.4,38.4,40.1,45.1,45.5,53.4,55.9,57.5,70.4,71.4$, 130.0, 148.5, 164.1, 174.60, 174.62, 176.4, 180.9. MS (FAB): $m / z 611\left(\mathrm{MH}^{+}\right)$. HRMS (FAB) calcd for $\mathrm{C}_{30} \mathrm{H}_{51} \mathrm{~N}_{4} \mathrm{O}_{7} \mathrm{~S}(\mathrm{M}+\mathrm{H})$ : 611.3480. Found: 611.3478.

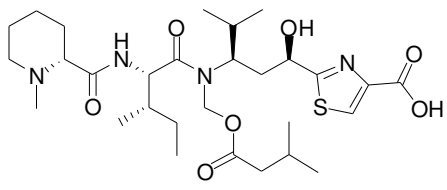

Compound 15. $\mathrm{Me}_{3} \mathrm{SnOH}(63.0 \mathrm{mg}, 0.350 \mathrm{mmol})$ was added to a $0.02 \mathrm{M}$ solution of methyl ester $14(26.7 \mathrm{mg}, 0.0437 \mathrm{mmol})$ in dichloroethane $(2.19 \mathrm{~mL})$, and the reaction mixture was heated to $60^{\circ} \mathrm{C}$ for $20 \mathrm{~h}$. The mixture was concentrated under reduced pressure. Column chromatography (100 mL of $100 \% \mathrm{CH}_{2} \mathrm{Cl}_{2}, 100 \mathrm{~mL}$ of $90: 10$ $\mathrm{CH}_{2} \mathrm{Cl}_{2} / \mathrm{MeOH}, 100 \mathrm{~mL}$ of 90:10:1 $\mathrm{CH}_{2} \mathrm{Cl}_{2} / \mathrm{MeOH} / \mathrm{NH}_{4} \mathrm{OH}, 100 \mathrm{~mL}$ 80:20:1 
$\left.\mathrm{CH}_{2} \mathrm{Cl}_{2} / \mathrm{MeOH} / \mathrm{NH}_{4} \mathrm{OH}\right)$ afforded $17.4 \mathrm{mg}(67 \%)$ of $\mathbf{1 5} \cdot[\alpha]^{23}{ }_{\mathrm{D}}=-2.00^{\circ}(c=1, \mathrm{MeOH})$. IR: 1585, 1651, 1738, 2964, 3132, 3202, $3424 \mathrm{~cm}^{-1} .{ }^{1} \mathrm{H}$ NMR (500 MHz, MeOD): $\delta$ 0.87-0.97 (m, 18H), $1.06(\mathrm{~m}, 2 \mathrm{H}), 1.16-1.30(\mathrm{~m}, 2 \mathrm{H}), 1.52-1.64(\mathrm{~m}, 2 \mathrm{H}), 1.70-1.78(\mathrm{~m}$, 2H), 1.82-1.88 (m, 2H), 1.92-2.12 (m, 5H), 2.23-2.33 (m, 2H), $2.64(\mathrm{~s}, 3 \mathrm{H}), 2.85-2.98(\mathrm{~m}$, 1H), 3.37-3.39 (m, 1H), 4.61-4.69 (m, 1H), 4.72-4.77 (m, 1H), 5.45 (d, 1H, $J=12.2 \mathrm{~Hz})$, $6.03(\mathrm{~d}, 1 \mathrm{H}, J=12.8 \mathrm{~Hz}), 7.99$ (s, 1H). ${ }^{13} \mathrm{C}$ NMR (125 MHz, MeOD): $\delta$ 11.7, 17.1, 21.6, 23.2, 23.56, 23.59, 23.61, 24.8, 26.5, 27.4, 27.6, 31.1, 38.5, 40.1, 44.0, 45.0, 57.08, 57.1, 68.9, 70.6, 130.0, 149.3, 165.1, 170.2, 174.7, 180.5, 180.6. MS (FAB): $m / z 597\left(\mathrm{MH}^{+}\right)$. HRMS (FAB) calcd for $\mathrm{C}_{29} \mathrm{H}_{49} \mathrm{~N}_{4} \mathrm{O}_{7} \mathrm{~S}(\mathrm{M}+\mathrm{H})$ : 597.3308. Found: 597.3322.

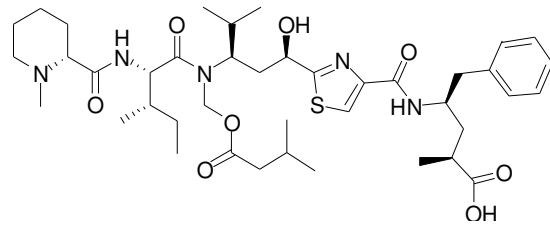

Compound 16. Acid $15(50.0 \mathrm{mg}, 0.0838 \mathrm{mmol})$ was added to a $0.2 \mathrm{M}$ solution of pentafluorophenol (23.0 $\mathrm{mg}, 0.125 \mathrm{mmol})$ and DIC (14.4 $\mu \mathrm{L}, 0.0919 \mathrm{mmol})$ in $\mathrm{CH}_{2} \mathrm{Cl}_{2}$ at $0{ }^{\circ} \mathrm{C}$. The reaction mixture was warmed to $\mathrm{rt}$, stirred for $24 \mathrm{~h}$, and concentrated under reduced pressure. EtOAc $(10 \mathrm{~mL})$ was added, and the crude product was filtered, with rinsing of the reaction vessel with EtOAc. The filtrate was concentrated under reduced pressure, and the crude material was used without further purification. DMF $(0.335 \mathrm{~mL}$, $0.25 \mathrm{M}$ ) was added to the crude product, followed by the hydrochloride salt of tubuphenylalanine (4) $(61.0 \mathrm{mg}, 0.250 \mathrm{mmol})$ and diisopropylethylamine $(73.0 \mu \mathrm{L}, 0.419$ mmol). The reaction mixture was stirred for $24 \mathrm{~h}$ at $\mathrm{rt}$, and DMF was removed under vacuum. HPFC purification (100:0 to $90: 10 \mathrm{CH}_{2} \mathrm{Cl}_{2}: \mathrm{MeOH}$ ) afforded $32.3 \mathrm{mg}(50 \%)$ of 15. The mixed fractions were repurified two more times via HPFC purification (100:0 to 
90:10 $\left.\mathrm{CH}_{2} \mathrm{Cl}_{2}: \mathrm{MeOH}\right)$ to afford a total of $56.0 \mathrm{mg}(85 \%)$ of $15 .[\alpha]^{23}{ }_{\mathrm{D}}=-3.90^{\circ}(c=1$, MeOH). IR: 1398, 1409, 1626, 2905, 2983, 3199, 3407, $3653 \mathrm{~cm}^{-1} .{ }^{1} \mathrm{H}$ NMR (500 MHz, MeOD): $\delta$ 0.85-0.93 (m, 12H), $1.00(\mathrm{~d}, 3 \mathrm{H}, J=7.3 \mathrm{~Hz}), 1.03(\mathrm{~d}, 3 \mathrm{H}, J=6.4 \mathrm{~Hz}), 1.18(\mathrm{~d}$, $3 \mathrm{H}, J=7.0 \mathrm{~Hz}), 1.21-1.32(\mathrm{~m}, 3 \mathrm{H}), 1.37-1.44(\mathrm{~m}, 1 \mathrm{H}), 1.60-1.69(\mathrm{~m}, 4 \mathrm{H}), 1.72-1.80(\mathrm{~m}$, 2H), 1.85-1.89 (m, 1H), 1.99-2.15 (m, 6H), $2.35(\mathrm{~s}, 3 \mathrm{H}), 2.39-2.46(\mathrm{~m}, 2 \mathrm{H}), 2.53-2.60$ (m, 1H), 2.88-2.96 (m, 2H), 3.00-3.07 (m, 1H), $3.12(\mathrm{~d}, 1 \mathrm{H}, J=12.5 \mathrm{~Hz}), 4.33-4.39(\mathrm{~m}, 1 \mathrm{H})$, $4.61(\mathrm{~d}, 1 \mathrm{H}, J=9.8 \mathrm{~Hz}), 4.76(\mathrm{~d}, 1 \mathrm{H}, J=10.1 \mathrm{~Hz}), 5.60(\mathrm{~d}, 1 \mathrm{H}, J=12.5 \mathrm{~Hz}), 6.11(\mathrm{~d}, 1 \mathrm{H}$, $J=12.4 \mathrm{~Hz}), 7.13-7.17(\mathrm{~m} \mathrm{1H}), 7.20-7.24(\mathrm{~m}, 4 \mathrm{H}), 8.02(\mathrm{~s}, 1 \mathrm{H}) .{ }^{13} \mathrm{C} \mathrm{NMR}(125 \mathrm{MHz}$, MeOD): $\delta 11.6,17.1,19.6,21.5,23.67,23.69,24.4,26.22,26.24,26.7,27.7,27.8,31.6$, $31.9,38.5,39.3,39.6,42.9,44.9,45.5,51.8,56.6,57.3,70.3,70.4,125.4,128.3,130.1$, 130.2, 130.8, 131.4, 140.4, 151.6, 163.9, 174.2, 179.87, 179.9. MS (FAB): $m / z 786$ $\left(\mathrm{MH}^{+}\right)$. HRMS (FAB) calcd for $\mathrm{C}_{41} \mathrm{H}_{64} \mathrm{~N}_{5} \mathrm{O}_{8} \mathrm{~S}(\mathrm{M}+\mathrm{H})$ : 786.4477. Found: 786.4476.

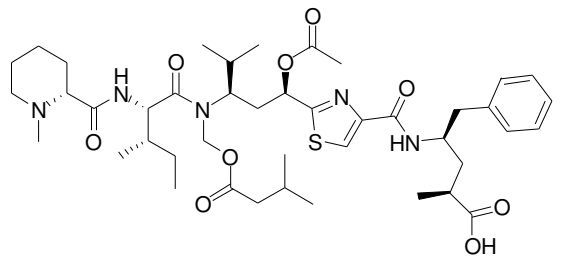

Tubulysin D (1). A $0.1 \mathrm{M}$ solution of $16(35.0 \mathrm{mg}, 0.0445 \mathrm{mmol})$ in pyridine $(0.445$ $\mathrm{mL})$ was cooled to $0{ }^{\circ} \mathrm{C}$, and acetic anhydride $(33.6 \mu \mathrm{L}, 0.356 \mathrm{mmol})$ was added. The reaction mixture was allowed to warm to rt over $2 \mathrm{~h}$ and was stirred at $\mathrm{rt}$ for $24 \mathrm{~h}$. The reaction mixture was then cooled to $0{ }^{\circ} \mathrm{C}$, and a $1: 1$ mixture of dioxane/water $(1.61 \mathrm{~mL})$ was added. The mixture was allowed to warm to $\mathrm{rt}$, and was stirred for $12 \mathrm{~h}$ at $\mathrm{rt}$. The solvent was removed under reduced pressure. Column chromatography $\left(100 \% \mathrm{CH}_{2} \mathrm{Cl}_{2}\right.$ to $10 \% \mathrm{MeOH} / \mathrm{CH}_{2} \mathrm{Cl}_{2}$ ) afforded $30.0 \mathrm{mg}(82 \%)$ of $\mathbf{1}$ as an amorphous solid. $[\alpha]^{23}=$ 
$+11.1^{\circ}(c=0.3, \mathrm{MeOH})\left(\mathrm{Lit}^{5}[\alpha]^{22}{ }_{\mathrm{D}}=+12.8^{\circ}(c=4, \mathrm{MeOH})\right.$. IR: $1547,1662,1738$, 2872, 2930, 2968, $3314 \mathrm{~cm}^{-1} .{ }^{1} \mathrm{H}$ NMR (500 MHz, MeOD): $\delta 0.82(\mathrm{~d}, 3 \mathrm{H}, J=6.25 \mathrm{~Hz})$, 0.85-0.92 (m, 9H), $0.98(\mathrm{~d}, 3 \mathrm{H}, J=7.02 \mathrm{~Hz}), 1.05(\mathrm{~d}, 3 \mathrm{H}, J=6.41 \mathrm{~Hz}), 1.17(\mathrm{~d}, 3 \mathrm{H}, J=$ $7.02 \mathrm{~Hz}), 1.39(\mathrm{~m}, 1 \mathrm{H}), 1.60-1.70(\mathrm{~m}, 5 \mathrm{H}), 1.78-1.87$ (m, 3H), 2.01 (m, 4H), 2.09-2.13

(m, 1H), 2.16 (s, 3H), 2.33 (brs, 5H), 2.45-2.55 (m, 2H), 2.88-2.94 (m, 3H), $3.07(\mathrm{~m}, 1 \mathrm{H})$, $4.36(\mathrm{~m}, 2 \mathrm{H}), 4.63(\mathrm{~d}, 2 \mathrm{H}, J=9.0 \mathrm{~Hz}), 5.46(\mathrm{~d}, 1 \mathrm{H}, J=12.0 \mathrm{~Hz}), 5.87(\mathrm{~d}, 1 \mathrm{H}, J=11.0$ Hz), $6.10(\mathrm{~d}, 1 \mathrm{H}, J=11.0 \mathrm{~Hz}), 7.15(\mathrm{~m}, 1 \mathrm{H}), 7.23(\mathrm{~m}, 4 \mathrm{H}), 8.10(\mathrm{~s}, 1 \mathrm{H}) . \mathrm{MS}(\mathrm{ESI}): \mathrm{m} / \mathrm{z}$ $828\left(\mathrm{MH}^{+}\right)$. HRMS (FAB) calcd for $\mathrm{C}_{43} \mathrm{H}_{66} \mathrm{~N}_{5} \mathrm{O}_{9} \mathrm{~S}(\mathrm{M}+\mathrm{H})$ : 828.4597. Found: 828.4581. For spectral comparison of the synthetic sample of tubulysin $\mathrm{D}$ in (MeOD and DMSO) to the sample isolated from nature, please see the tables of values below.

\section{Table of ${ }^{13} \mathrm{C}$ spectral data in MeOD.}

\begin{tabular}{|c|c|}
\hline Natural $(\mathbf{7 5} \mathbf{~ M H z}) \delta^{5}$ & Synthetic $(125 \mathrm{MHz}) \delta$ \\
\hline 180.7 & 181.1 \\
\hline 176.3 & 176.4 \\
\hline 173.3 & 173.8 \\
\hline 172.9 & 173.2 \\
\hline 171.9 & 171.9 \\
\hline 170.8 & 170.8 \\
\hline 162.7 & 162.7 \\
\hline 150.8 & 150.8 \\
\hline 139.6 & 139.6 \\
\hline 130.5 & 130.5 \\
\hline 129.3 & 129.3 \\
\hline 127.4 & 127.4 \\
\hline 125.5 & 125.5 \\
\hline 70.8 & 70.7 \\
\hline 69.3 & 69.7 \\
\hline 56.4 & 56.5 \\
\hline 55.4 & 55.3 \\
\hline 51.0 & 51.1 \\
\hline 44.3 & 44.4 \\
\hline 43.9 & 44.2 \\
\hline 42.1 & 42.0 \\
\hline
\end{tabular}

\begin{tabular}{|c|c|}
\hline Natural $\mathbf{( \mathbf { 7 5 ~ } \mathbf { ~ H z } )} \boldsymbol{\delta}$ & Synthetic $\mathbf{( \mathbf { 1 2 5 } \mathbf { ~ M H z } )} \boldsymbol{\delta}$ \\
\hline 39.4 & 39.6 \\
\hline 38.4 & 38.8 \\
\hline 37.4 & 37.4 \\
\hline 35.8 & 35.8 \\
\hline 32.2 & 32.3 \\
\hline 31.0 & 31.2 \\
\hline 26.8 & 26.8 \\
\hline 25.5 & 25.6 \\
\hline 25.2 & 25.5 \\
\hline 23.4 & 23.7 \\
\hline 22.8 & 22.8 \\
\hline 20.8 & 20.8 \\
\hline 20.7 & 20.7 \\
\hline 20.4 & 20.4 \\
\hline 18.7 & 18.9 \\
\hline 16.4 & 16.4 \\
\hline 10.8 & 10.7 \\
\hline
\end{tabular}


Table of ${ }^{1} \mathrm{H}$ NMR and ${ }^{13} \mathrm{C}$ NMR spectral data in DMSO.

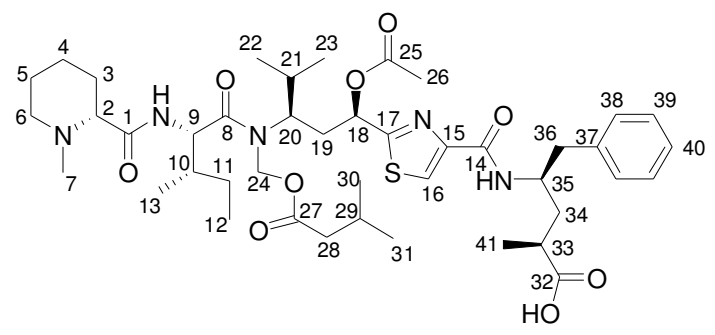

\begin{tabular}{|c|c|c|c|c|c|}
\hline $\mathrm{H}$ atoms & 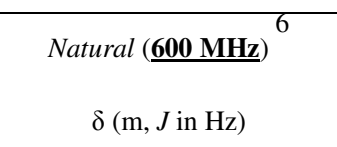 & $\begin{array}{c}\text { Synthetic } \underline{(\underline{500 ~ \mathbf{~ M H z}})} \\
\delta(\mathrm{m}, J \text { in } \mathrm{Hz})\end{array}$ & $\mathrm{C}$ atoms & $\begin{array}{c}\text { Natural } \\
(150 \mathrm{MHz}) \delta\end{array}$ & $\begin{array}{c}\text { Synthetic } \\
(125 \mathrm{MHz}) \delta\end{array}$ \\
\hline $\mathrm{H} 2$ & $2.46(\mathrm{dd}, 10.4,3.1) 1 \mathrm{H}$ & $2.46(\mathrm{~m}) 1 \mathrm{H}$ & $\mathrm{C} 1$ & 172.8 & 172.8 \\
\hline $\mathrm{H} 3 \mathrm{a}$ & $1.37(\mathrm{~m}) 1 \mathrm{H}$ & $1.37(\mathrm{~m}) 1 \mathrm{H}$ & $\mathrm{C} 2$ & 68.1 & 68.1 \\
\hline $\mathrm{H} 3 \mathrm{~b}$ & $1.57(\mathrm{~m}) 1 \mathrm{H}$ & $1.57(\mathrm{~m}) 1 \mathrm{H}$ & $\mathrm{C} 3$ & 24.8 & 24.8 \\
\hline $\mathrm{H} 4 \mathrm{a}$ & $1.16(\mathrm{qt}, 12.3,3.6) 1 \mathrm{H}$ & $1.16(\mathrm{~m}) 1 \mathrm{H}$ & $\mathrm{C} 4$ & 22.8 & 22.8 \\
\hline $\mathrm{H} 4 \mathrm{~b}$ & $1.63(\mathrm{~m}) 1 \mathrm{H}$ & $1.63(\mathrm{~m}) 1 \mathrm{H}$ & $\mathrm{C} 5$ & 29.6 & 29.6 \\
\hline $\mathrm{H} 5 \mathrm{a}$ & $1.42(\mathrm{qt}, 12.5,3.3) 1 \mathrm{H}$ & $1.42(\mathrm{~m}) 1 \mathrm{H}$ & C6 & 54.7 & 54.69 \\
\hline $\mathrm{H} 5 \mathrm{~b}$ & $1.55(\mathrm{~m}) 1 \mathrm{H}$ & $1.55(\mathrm{~m}) 1 \mathrm{H}$ & $\mathrm{C} 7$ & 43.8 & 43.8 \\
\hline H6a & $1.93(\mathrm{~m}) 1 \mathrm{H}$ & $1.91(\mathrm{~m}) 1 \mathrm{H}$ & $\mathrm{C} 8$ & 174.2 & 174.2 \\
\hline $\mathrm{H} 6 \mathrm{~b}$ & $2.82(\mathrm{dt}, 11.5,3.5) 1 \mathrm{H}$ & $2.79(\mathrm{~m}) 1 \mathrm{H}$ & C9 & 52.6 & 52.6 \\
\hline $\mathrm{H} 7$ & $2.04(\mathrm{~s}) 3 \mathrm{H}$ & $2.02(\mathrm{~s}) 3 \mathrm{H}$ & $\mathrm{C} 10$ & 35.1 & 35.1 \\
\hline H9 & $4.42(\mathrm{t}, 9.1) 1 \mathrm{H}$ & $4.39(\mathrm{t}, 9.0) 1 \mathrm{H}$ & $\mathrm{C} 11$ & 24.1 & 24.1 \\
\hline 9-NH & $7.92(\mathrm{~d}, 8.8) 1 \mathrm{H}$ & $7.95(\mathrm{~d}, 8.4) 1 \mathrm{H}$ & $\mathrm{C} 12$ & 10.1 & 10.0 \\
\hline $\mathrm{H} 10$ & $1.93(\mathrm{~m}) 1 \mathrm{H}$ & $1.91(\mathrm{~m}) 1 \mathrm{H}$ & $\mathrm{C} 13$ & 15.3 & 15.3 \\
\hline H11a & $1.09(\mathrm{~m}) 1 \mathrm{H}$ & $1.07(\mathrm{~m}) 1 \mathrm{H}$ & $\mathrm{C} 14$ & 159.7 & 159.8 \\
\hline $\mathrm{H} 11 \mathrm{~b}$ & $1.48(\mathrm{ddd}, 13.4,7.5,2.8) 1 \mathrm{H}$ & $1.48(\mathrm{~m}) 1 \mathrm{H}$ & $\mathrm{C} 15$ & 149.8 & 149.7 \\
\hline $\mathrm{H} 12$ & $0.80(\mathrm{t}, 7.7) 3 \mathrm{H}$ & $0.80(\mathrm{~m}) 3 \mathrm{H}$ & $\mathrm{C} 16$ & 124.2 & 124.4 \\
\hline H13 & $0.83(\mathrm{~d}, 6.1) 3 \mathrm{H}$ & $0.82(\mathrm{~m}) 3 \mathrm{H}$ & $\mathrm{C} 17$ & 168.5 & 168.5 \\
\hline H16 & $8.18(\mathrm{~s}) 1 \mathrm{H}$ & $8.19(\mathrm{~s}) 1 \mathrm{H}$ & $\mathrm{C} 18$ & 68.9 & 68.9 \\
\hline $\mathrm{H} 18$ & $5.75(\mathrm{dd}, 11.3,2.2) 1 \mathrm{H}$ & $5.73(\mathrm{~d}, 11.5) 1 \mathrm{H}$ & C19 & 34.3 & 34.3 \\
\hline H19a & 2.15 (brs) $1 \mathrm{H}$ & $2.15(\mathrm{~m}) 1 \mathrm{H}$ & $\mathrm{C} 20$ & 55.8 & 54.71 \\
\hline $\mathrm{H} 19 \mathrm{~b}$ & $2.40(\mathrm{~m}) 1 \mathrm{H}$ & $2.35(\mathrm{~m}) 1 \mathrm{H}$ & $\mathrm{C} 21$ & 30.0 & 30.1 \\
\hline $\mathrm{H} 20$ & 4.38 (brs) $1 \mathrm{H}$ & 4.38 (brs) $1 \mathrm{H}$ & $\mathrm{C} 22$ & 19.3 & 19.3 \\
\hline
\end{tabular}




\begin{tabular}{|c|c|c|c|c|c|}
\hline $\mathrm{H} 21$ & 1.83 (brs) $1 \mathrm{H}$ & $1.81(\mathrm{~m}) 1 \mathrm{H}$ & $\mathrm{C} 23$ & 20.2 & 20.2 \\
\hline $\mathrm{H} 22$ & $0.68(\mathrm{~d}, 6.7) 3 \mathrm{H}$ & $0.66(\mathrm{~d}, 6.4) 3 \mathrm{H}$ & $\mathrm{C} 24$ & 68.9 & 68.9 \\
\hline $\mathrm{H} 23$ & $0.98(\mathrm{~d}, 6.5) 3 \mathrm{H}$ & $0.96(\mathrm{~d}, 6.6) 3 \mathrm{H}$ & $\mathrm{C} 25$ & 169.8 & 169.8 \\
\hline $\mathrm{H} 24 \mathrm{a}$ & $5.26(\mathrm{~d}, 12.1) 1 \mathrm{H}$ & $5.24(\mathrm{~d}, 12.0) 1 \mathrm{H}$ & $\mathrm{C} 26$ & 20.5 & 20.5 \\
\hline $\mathrm{H} 24 \mathrm{~b}$ & $6.20(\mathrm{brd}, 12.1) 1 \mathrm{H}$ & 6.19 (brd, 11.3) $1 \mathrm{H}$ & $\mathrm{C} 27$ & 171.3 & 171.3 \\
\hline $\mathrm{H} 26$ & $2.11(\mathrm{~s}) 3 \mathrm{H}$ & $2.09(\mathrm{~s}) 3 \mathrm{H}$ & $\mathrm{C} 28$ & 42.7 & 42.7 \\
\hline $\mathrm{H} 28 \mathrm{a}$ & $2.08(\mathrm{dd}, 14.9,7.1) 1 \mathrm{H}$ & $2.12(\mathrm{~m}) 1 \mathrm{H}$ & $\mathrm{C} 29$ & 25.0 & 25.0 \\
\hline $\mathrm{H} 28 \mathrm{~b}$ & $2.14(\mathrm{dd}, 14.9,7.2) 1 \mathrm{H}$ & $2.13(\mathrm{~m}) 1 \mathrm{H}$ & $\mathrm{C} 30$ & 22.1 & 22.0 \\
\hline $\mathrm{H} 29$ & $1.90(\mathrm{~m}) 1 \mathrm{H}$ & $1.91(\mathrm{~m}) 1 \mathrm{H}$ & $\mathrm{C} 31$ & 22.0 & 21.97 \\
\hline $\mathrm{H} 30$ & $0.82(\mathrm{~d}, 6.7) 3 \mathrm{H}$ & $0.80(\mathrm{~m}) 3 \mathrm{H}$ & $\mathrm{C} 32$ & 177.1 & 176.9 \\
\hline $\mathrm{H} 31$ & $0.81(\mathrm{~d}, 6.7) 3 \mathrm{H}$ & $0.79(\mathrm{~m}) 3 \mathrm{H}$ & $\mathrm{C} 33$ & 36.2 & 35.9 \\
\hline $\mathrm{H} 33$ & $2.38(\mathrm{~m}) 1 \mathrm{H}$ & $2.35(\mathrm{~m}) 1 \mathrm{H}$ & $\mathrm{C} 34$ & 37.6 & 37.7 \\
\hline $\mathrm{H} 34 \mathrm{a}$ & $1.52(\mathrm{ddd}, 13.6,10.6,4.9) 1 \mathrm{H}$ & $1.54(\mathrm{~m}) 1 \mathrm{H}$ & $\mathrm{C} 35$ & 49.0 & 48.7 \\
\hline $\mathrm{H} 34 \mathrm{~b}$ & $1.84(\mathrm{ddd}, 13.5,9.1,4.3) 1 \mathrm{H}$ & $1.81(\mathrm{~m}) 1 \mathrm{H}$ & $\mathrm{C} 36$ & 39.5 & under solvent peak \\
\hline $\mathrm{H} 35$ & $4.11(\mathrm{~m}) 1 \mathrm{H}$ & $4.16(\mathrm{~m}) 1 \mathrm{H}$ & $\mathrm{C} 37$ & 138.4 & 138.5 \\
\hline $35-\mathrm{NH}$ & $7.81(\mathrm{~d}, 9.0) 1 \mathrm{H}$ & 8.34 (brs) $1 \mathrm{H}$ & $\mathrm{C} 38$ & 129.0 & 129.1 \\
\hline $\mathrm{H} 36 \mathrm{a}$ & $2.67(\mathrm{dd}, 13.8,6.3) 1 \mathrm{H}$ & $2.75(\mathrm{~m}) 1 \mathrm{H}$ & $\mathrm{C} 39$ & 128.0 & 128.1 \\
\hline $\mathrm{H} 36 \mathrm{~b}$ & $2.73(\mathrm{dd}, 13.8,7.3) 1 \mathrm{H}$ & $2.79(\mathrm{~m}) 1 \mathrm{H}$ & $\mathrm{C} 40$ & 125.9 & 126.0 \\
\hline $\mathrm{H} 38-40$ & $7.20(\mathrm{~m}) 5 \mathrm{H}$ & $7.19(\mathrm{~m}) 5 \mathrm{H}$ & $\mathrm{C} 41$ & 18.0 & 18.0 \\
\hline $\mathrm{H} 41$ & $1.06(\mathrm{~d}, 7.0) 3 \mathrm{H}$ & $1.01(\mathrm{~d}, 7.0) 3 \mathrm{H}$ & & & \\
\hline
\end{tabular}

1. Liu, G.; Cogan, D. A.; Owens, T. D.; Tang, T. P.; Ellman, J. A. J. Org. Chem. 1999, $64,1278$.

2. Inami, K.; Shiba, T. Bull. Chem. Soc. Jpn. 1985, 58, 352.

3. Lundquist, J. T. IV.; Pelletier, J. C. Org. Lett. 2001, 3, 781.

4. Abe, T.; Kumagai, T. Carbapenem-3-carboxylic Acid Ester Derivatives. PCT Int. Appl. 1997, WO 97/21712.

5. The authors thank Prof. Sasse and Prof. Jansen for generously supplying ${ }^{1} \mathrm{H}$ NMR and ${ }^{13} \mathrm{C}$ NMR spectra of tubulysin D in MeOD.

6. Steinmetz, H.; Glaser, N.; Herdtweck, E.; Sasse, F.; Reichenbach, H.; Höfle, G. Angew. Chem. Int. Ed. 2004, 43, 4888. 

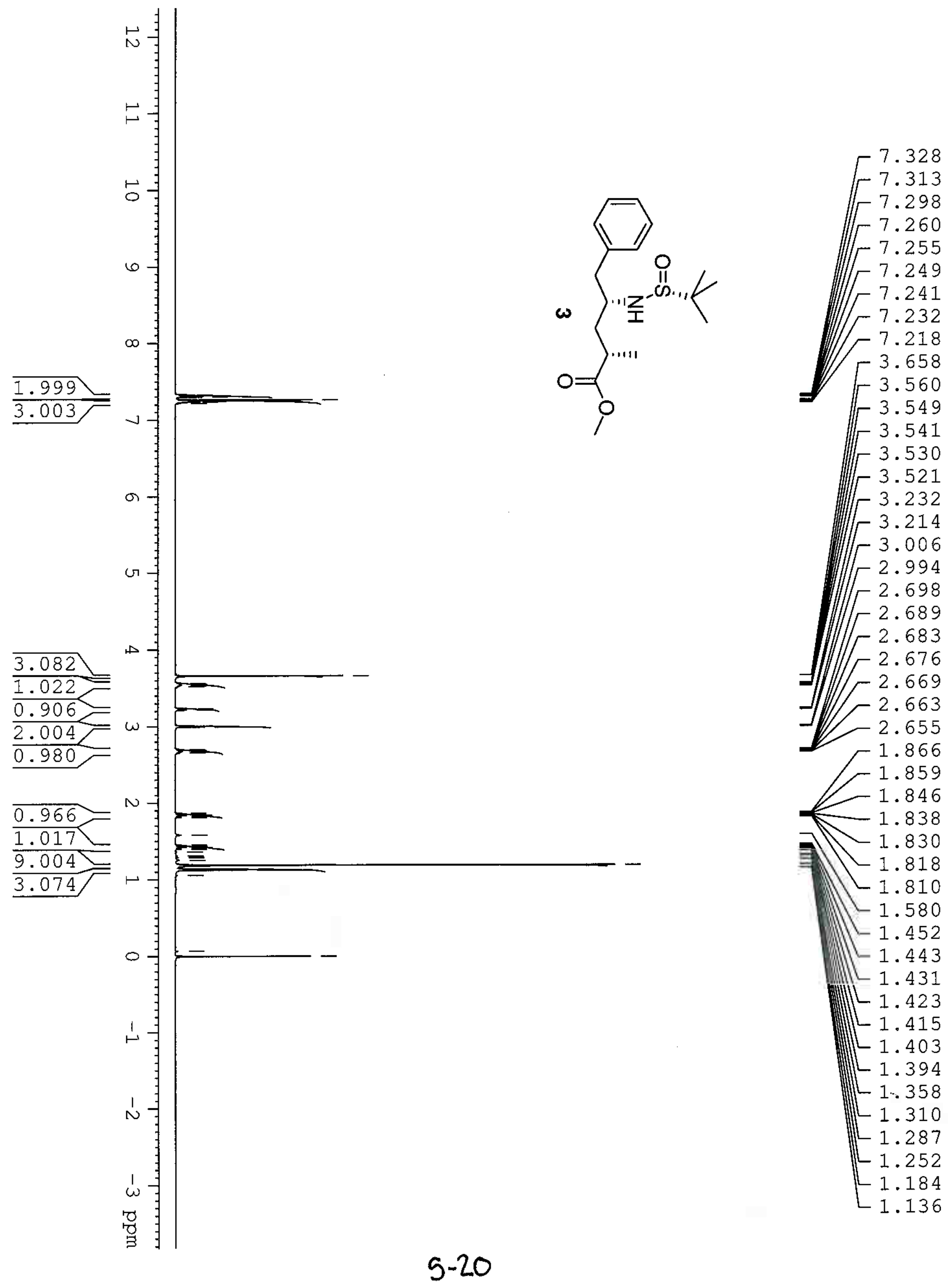


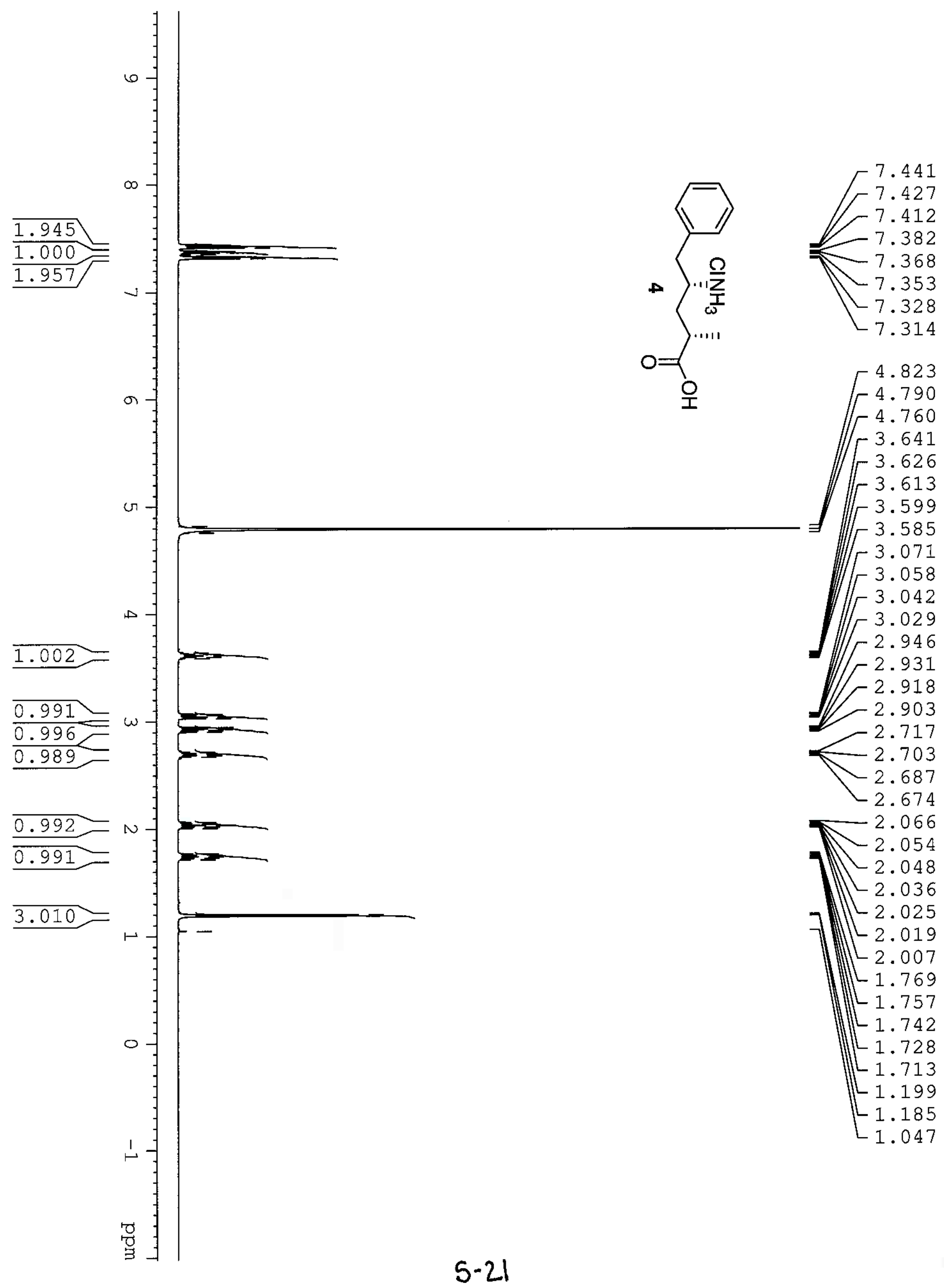



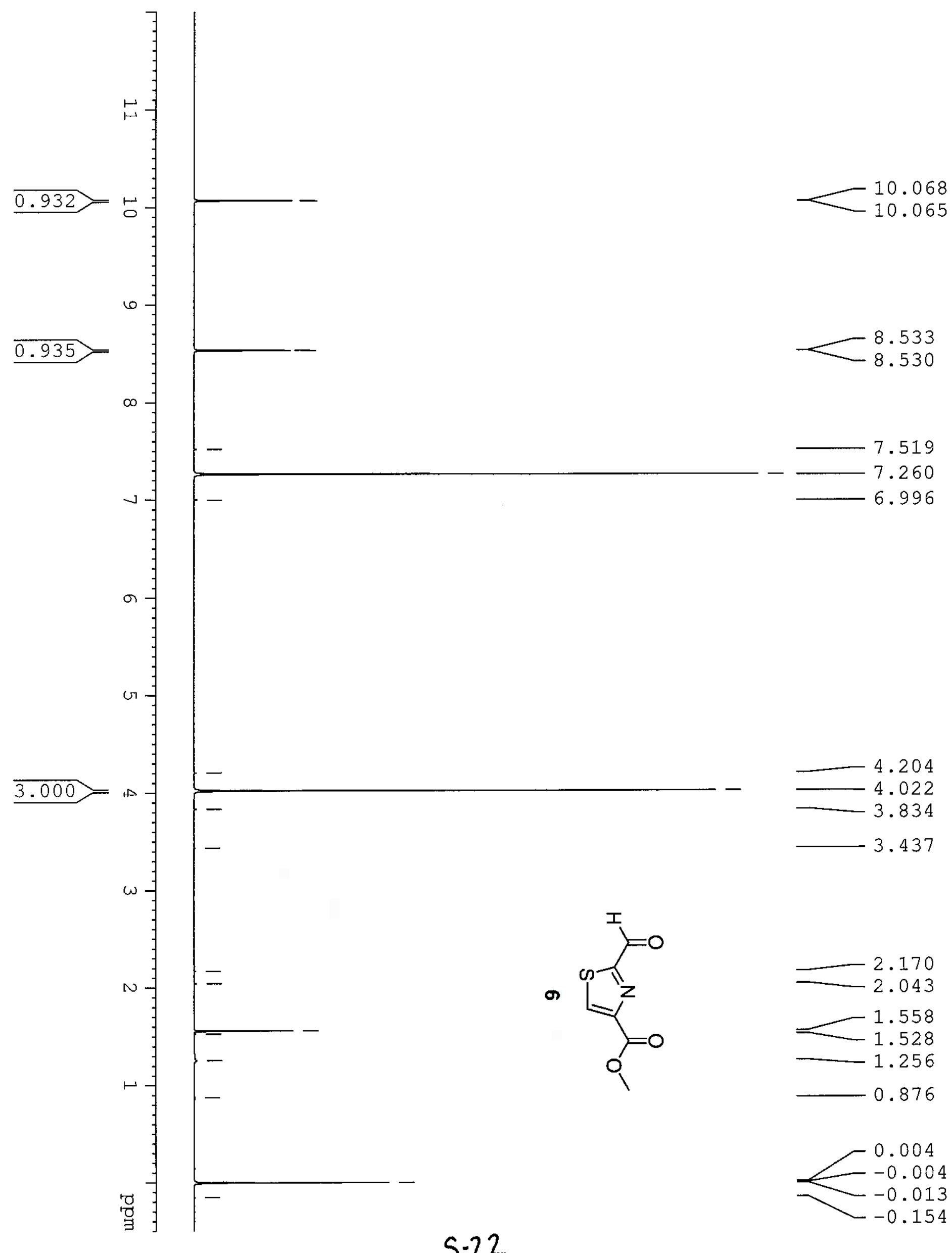

8.530

7.519

7.260

6.996

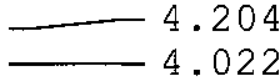

$-3.834$

3.437
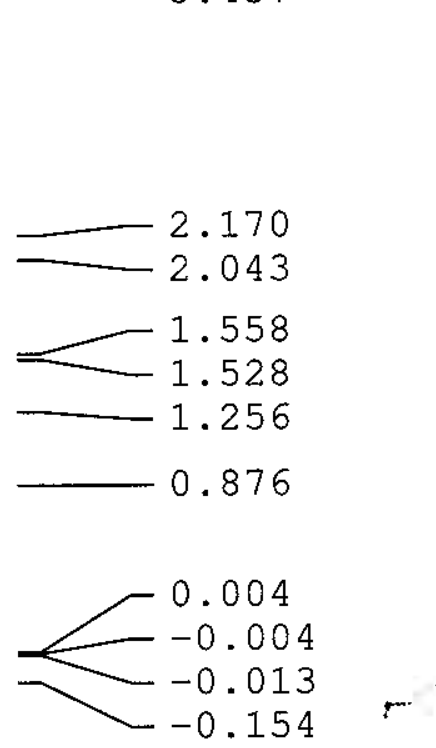

$5-22$ 

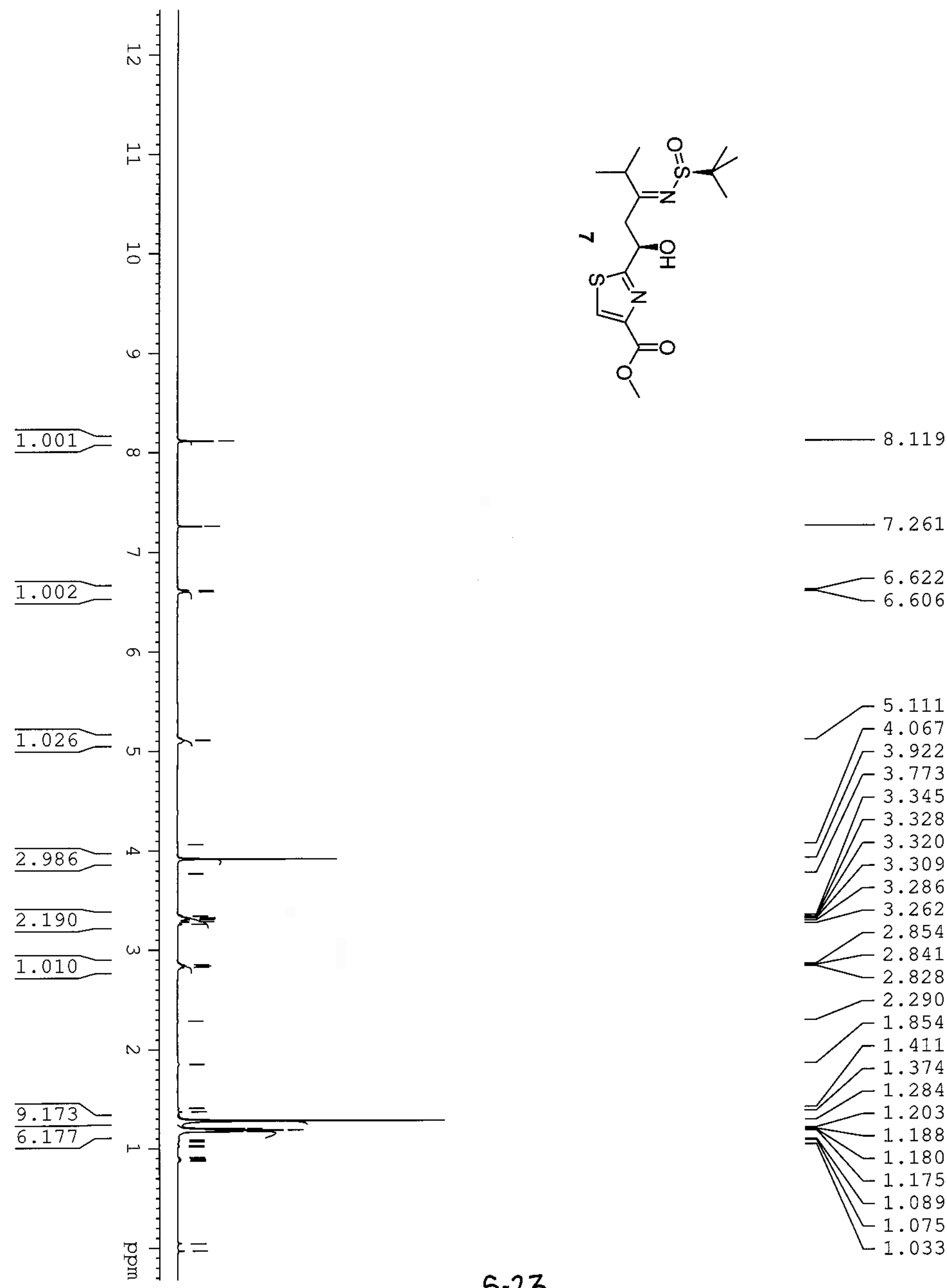

$-3.922$

$-3.773$

$-3.345$

$-3.328$

$\pi / 3.320$

$/ / F-3.309$

$-3.286$

2.262

$-2.854$

2.841
-2.828

-2.290
$-\quad 1.854$

$\gamma^{1.854} 1.411$

$-1.374$

$-1.284$

$=1.203$

$\approx-1.188$

$-1.180$

$\lfloor 1.175$

$\backslash 1.089$

$-1.075$

$\llcorner 1.033$ 


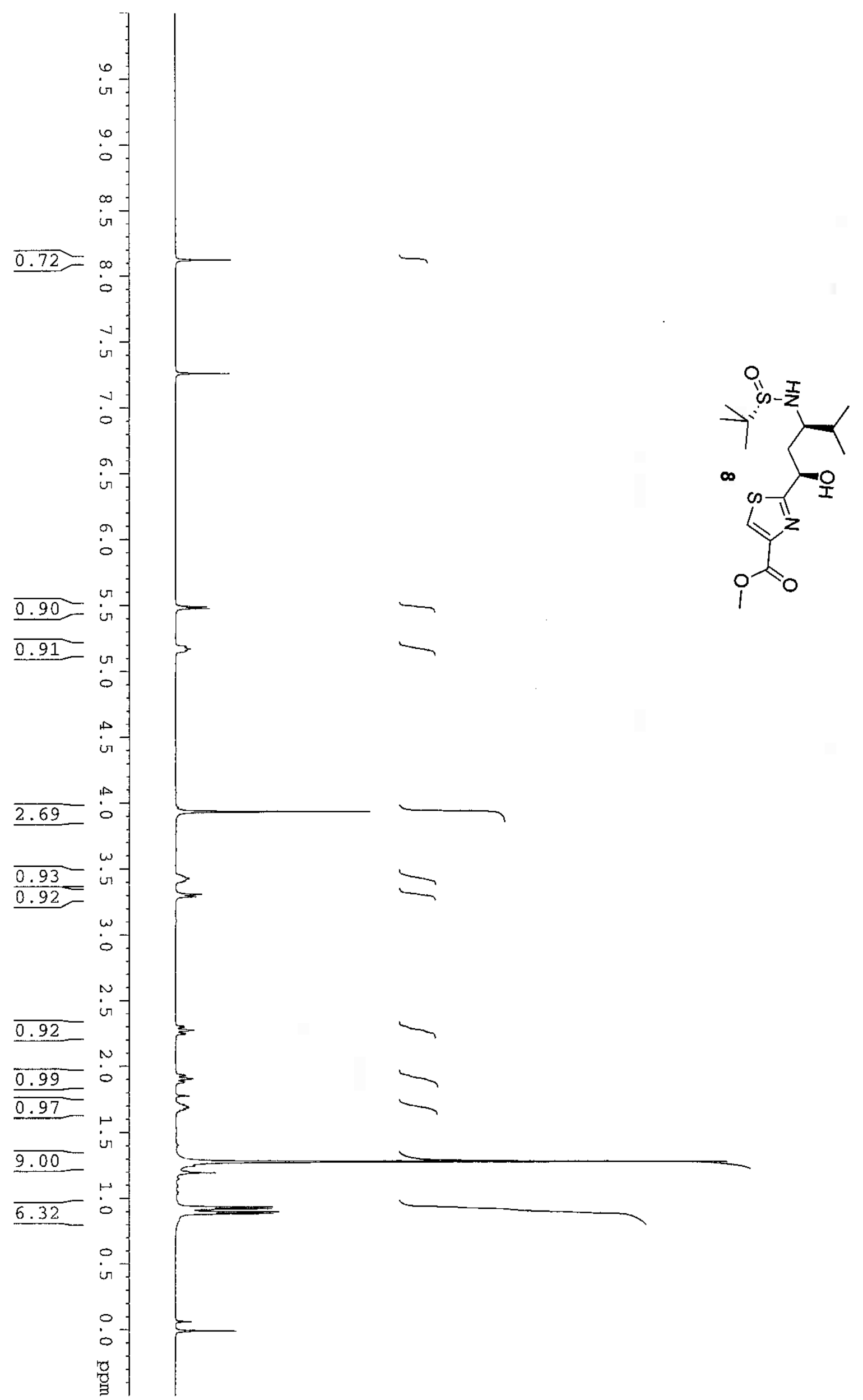

8.123

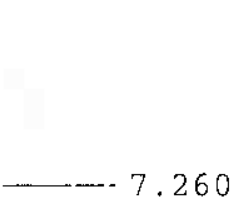

$-5.489$

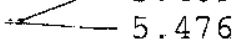

$-5.186$

$-5.170$

-. 5.155

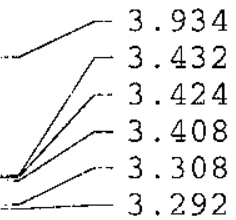

$-2.298$

$-2.274$

$/ 2.250$

1.935

$/ / r_{-1.928}$

$\| / r^{-1.905}$

1.882

1.776

1.710

$\because-1.696$

1.687

$\ldots \quad 1.674$

- $2 \begin{array}{r}1.404 \\ -1.278\end{array}$

h 1.195

1.148

M 1.103

$-1.049$

$-0.935$

$-0.921$

L 0.899

$-0.885$

0.061 


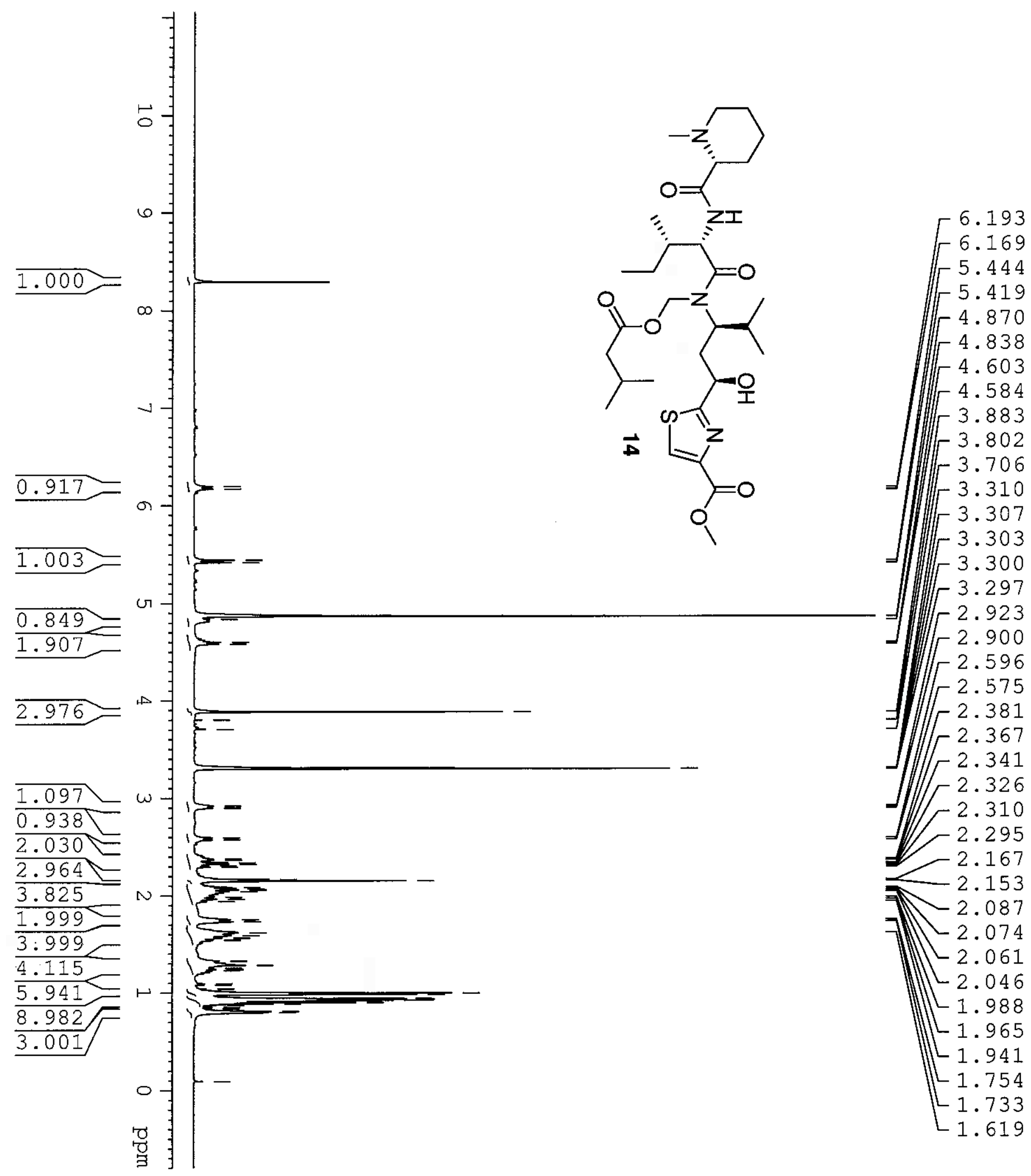




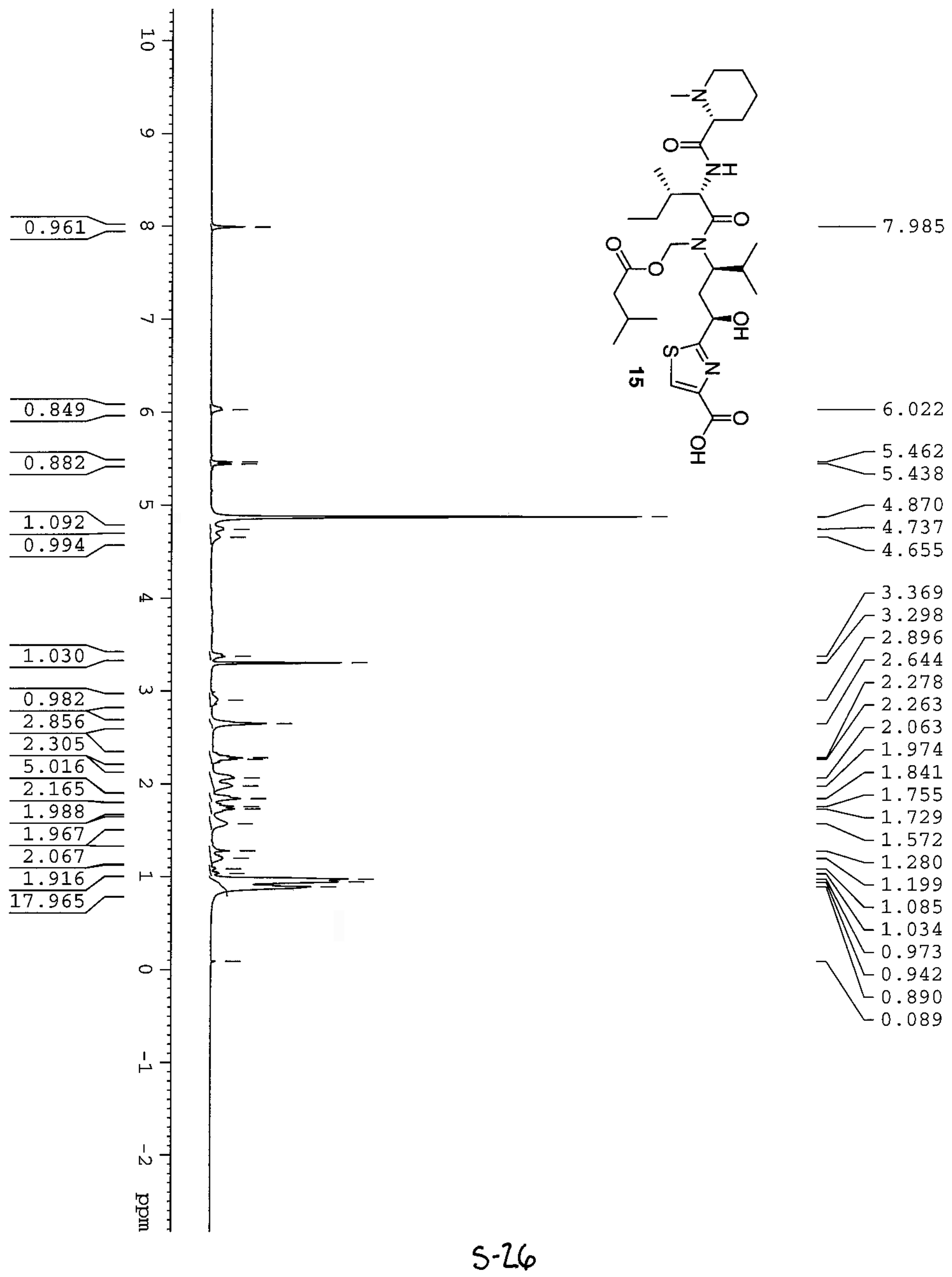




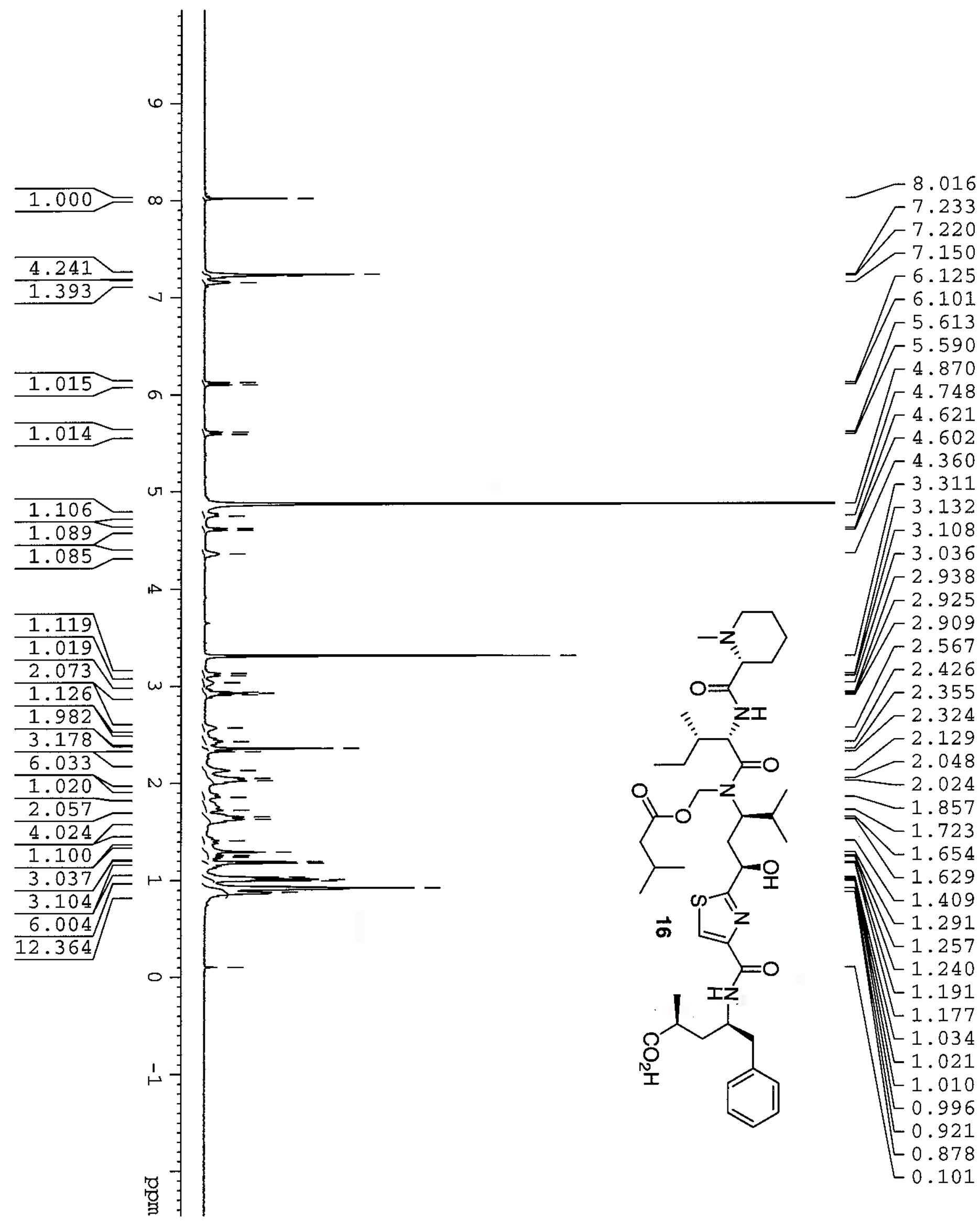




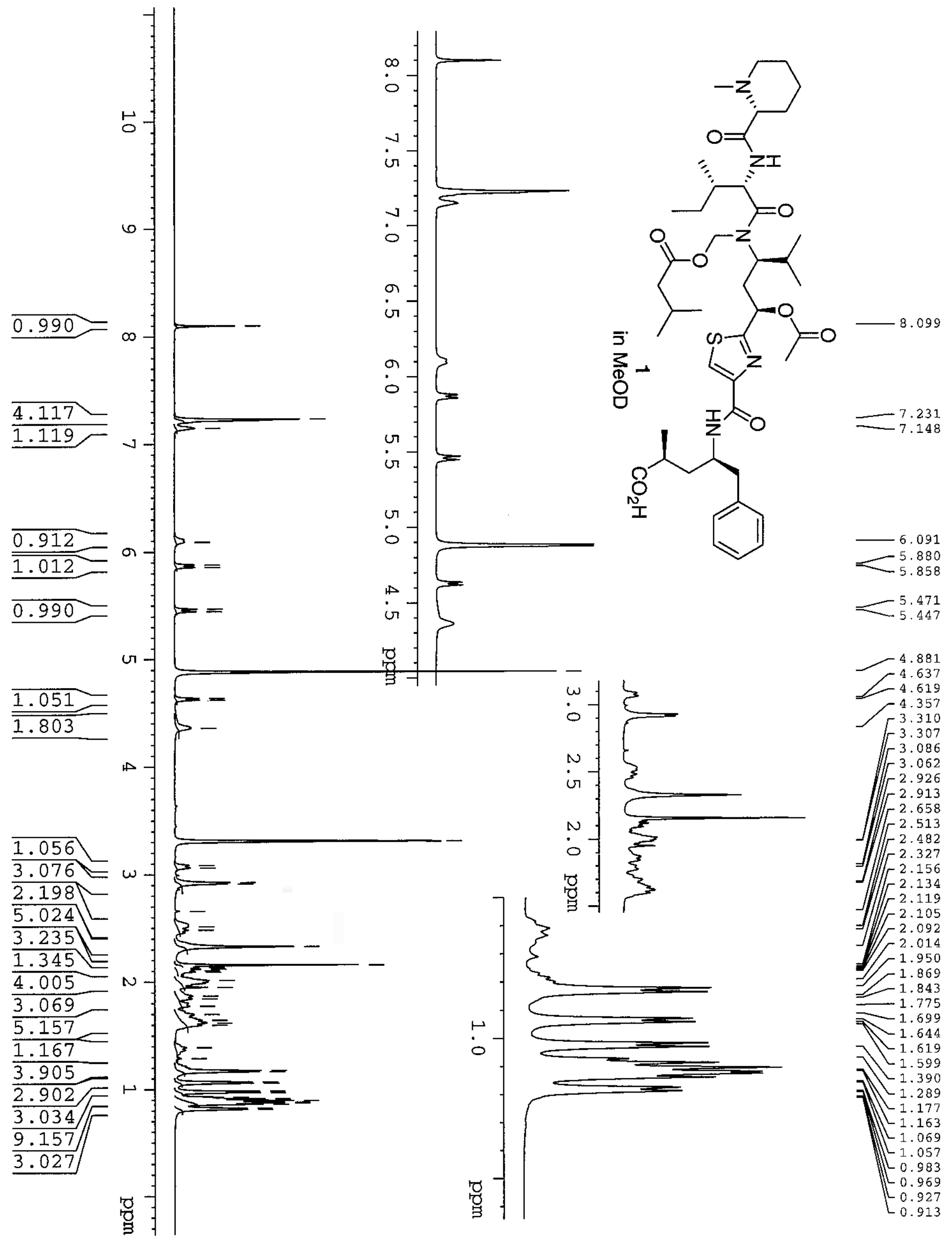




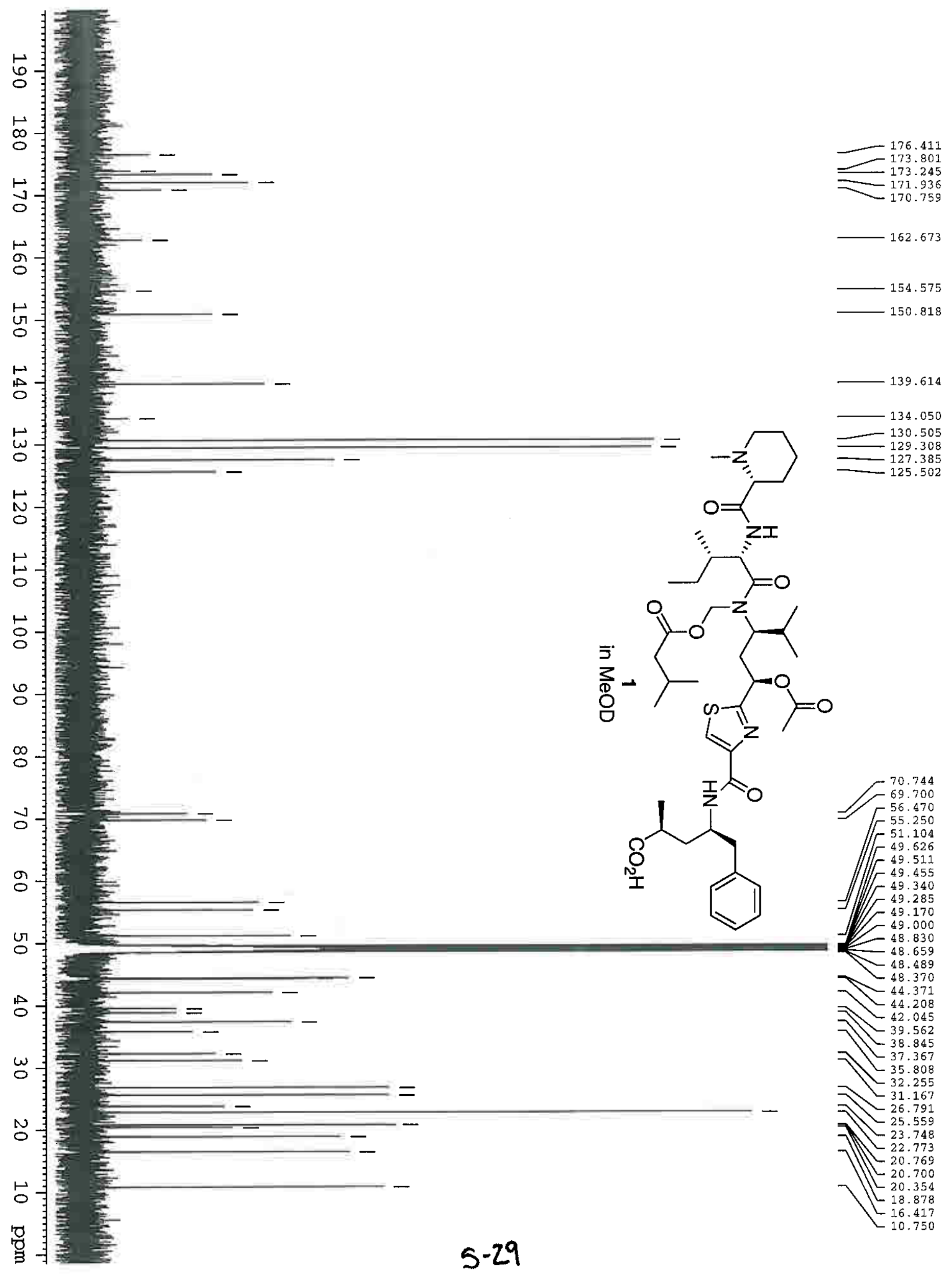




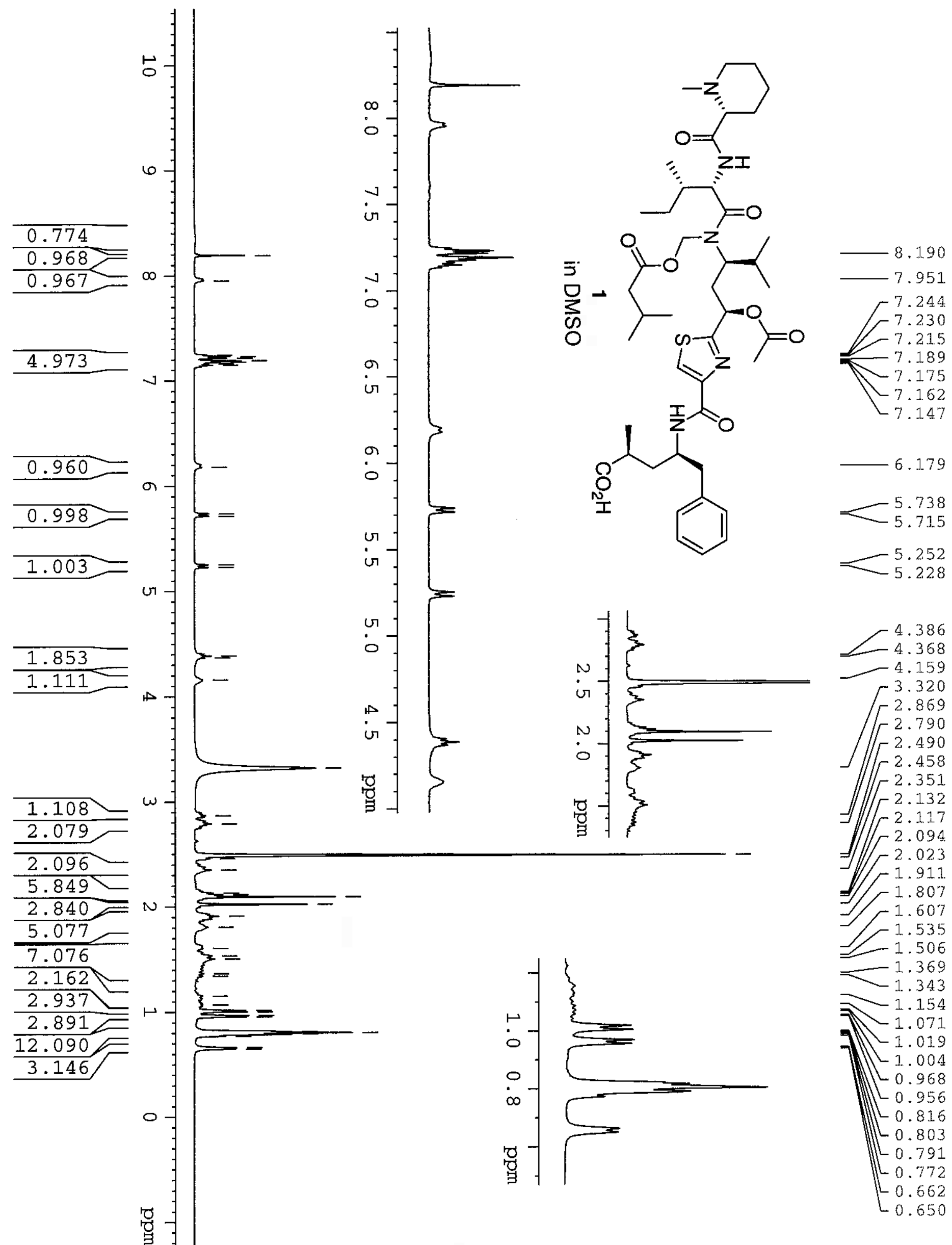



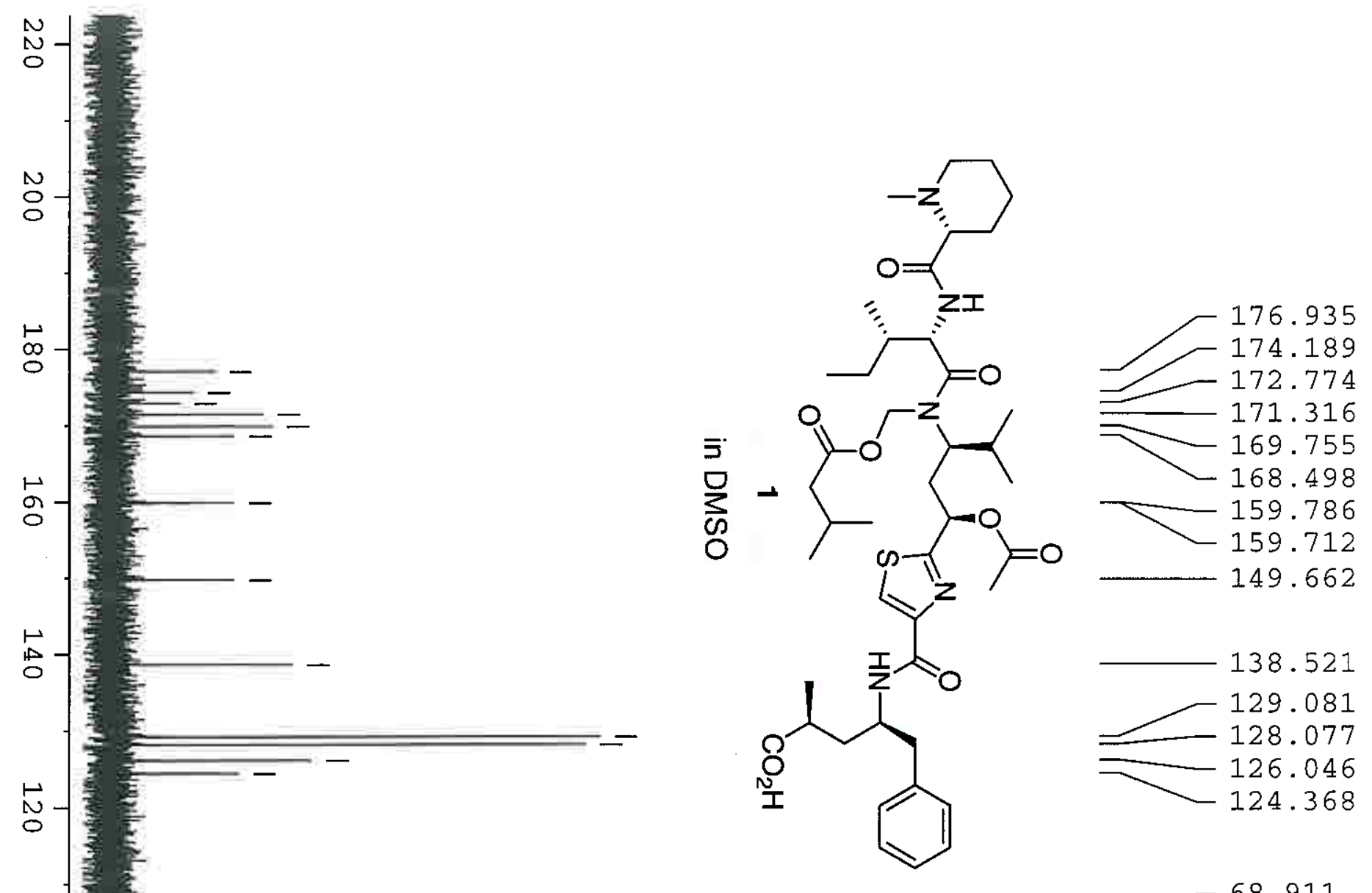

응

. 


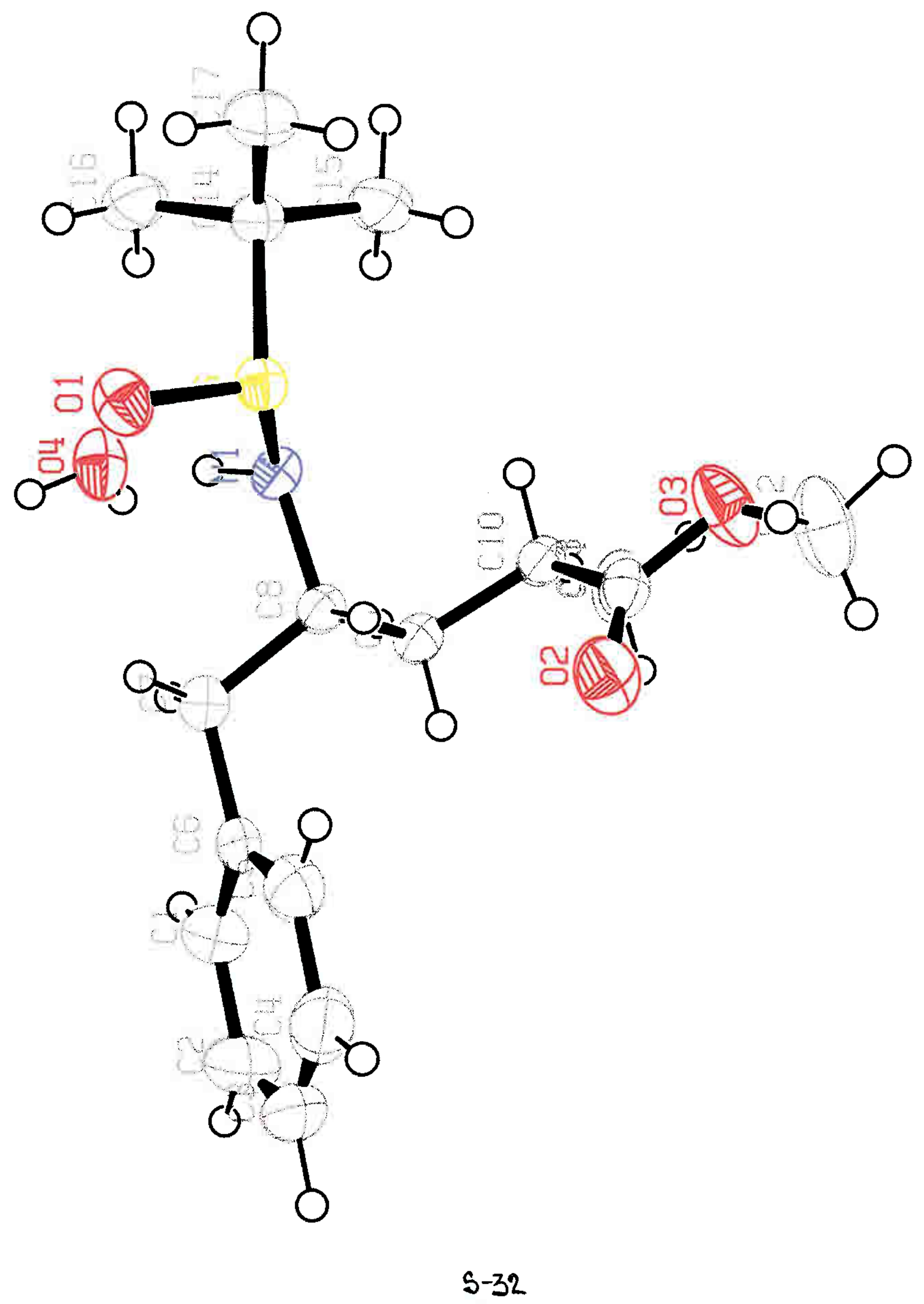




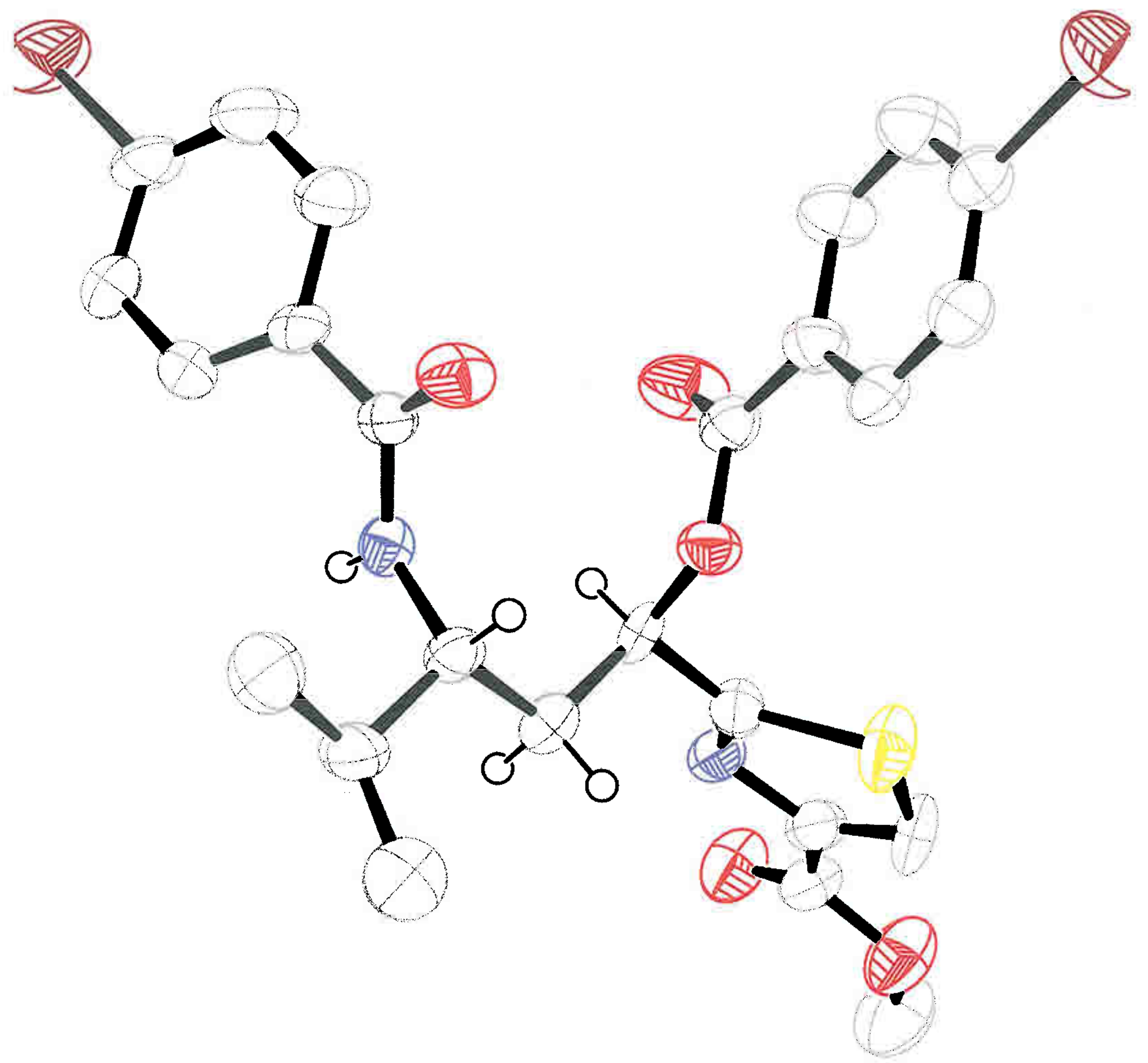

$5-33$ 